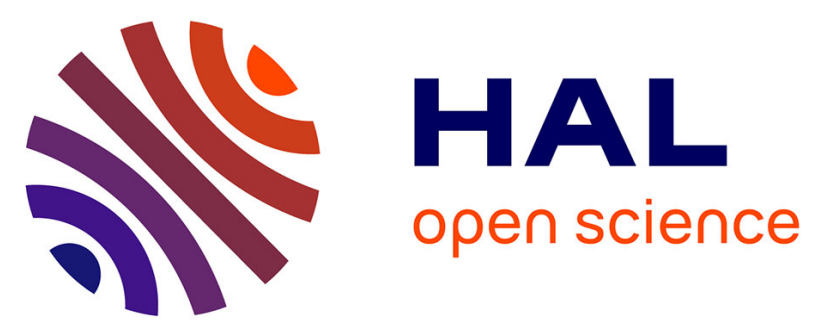

\title{
Proof of the equivalence of Tanizawa-Berkvens's and Cointe-van Daalen's formulations for the time derivative of the velocity potential for non-linear potential flow solvers
}

\author{
Lucas Letournel, Guillaume Ducrozet, Aurélien Babarit, Pierre Ferrant
}

\section{To cite this version:}

Lucas Letournel, Guillaume Ducrozet, Aurélien Babarit, Pierre Ferrant. Proof of the equivalence of Tanizawa-Berkvens's and Cointe-van Daalen's formulations for the time derivative of the velocity potential for non-linear potential flow solvers. Applied Ocean Research, 2017, 63, pp.184-199. 10.1016/j.apor.2017.01.010 . hal-01496149v2

\section{HAL Id: hal-01496149 \\ https://hal.science/hal-01496149v2}

Submitted on 13 Apr 2017

HAL is a multi-disciplinary open access archive for the deposit and dissemination of scientific research documents, whether they are published or not. The documents may come from teaching and research institutions in France or abroad, or from public or private research centers.
L'archive ouverte pluridisciplinaire HAL, est destinée au dépôt et à la diffusion de documents scientifiques de niveau recherche, publiés ou non, émanant des établissements d'enseignement et de recherche français ou étrangers, des laboratoires publics ou privés.

\section{(ㅇ)(1) $\$$}

Distributed under a Creative Commons Attribution - NonCommercial - NoDerivatives 44.0 


\title{
Proof of the equivalence of Tanizawa-Berkvens's and Cointe-van Daalen's formulations for the time derivative of the velocity potential for non-linear potential flow solvers.
}

\author{
LETOURNEL Lucas ${ }^{\star}$, DUCROZET Guillaume *, BABARIT Aurélien *, and \\ FERRANT Pierre *
}

^École Centrale de Nantes, LHEEA Lab., CNRS, Nantes, France

April 13, 2017

\begin{abstract}
Dealing with freely-floating bodies in the framework of non-linear potential flow theory may require solving Laplace's equation for the time derivative of the velocity potential. At present, there are two competing formulations for the body boundary condition. The first one was derived by Cointe Cointe(1989) in 2D. It was later extended to 3D by van Daalen $[\operatorname{van}$ Daalen(1993)]. The second formulation was derived by Tanizawa Tanizawa(1995)] in 2D. It was extended to 3D by Berkvens Berkvens(1998). In this paper, a proof is given that the Cointe-Van Daalen's and the Tanizawa-Berkvens' formulations are equivalent. It leads to a simplified version of Cointe-Van Daalen's formulation. The formulation is validated against the analytical solution for a moving sphere in an unbounded water domain.
\end{abstract}

\section{INTRODUCTION}

The first breakthrough for the simulation of nonlinear waves in time domain came from the Mixed Euler Lagrande (MEL) method, introduced by Longuet-Higgins and Cokelet [Longuet-Higgins and Cokelet(1976)]. Empowered by this approach, many numerical wave tanks (NWT) were developed, to simulate nonlinear wave propagation Xü, H Yue(1992)], plunging waves [Dommermuth and Yue(1987), Zhang et al.(1996)Zhang, Yue, and Tanis overturning waves Grilli et al.(2000)Grilli, Guyenne, and Dias in 2D or 3D. Simulations of wave diffraction on bodies with fixed or prescribed motions were also possible Ferrant(1998), Kashiwagi(1996)]. The accurate evaluation of the time derivative of the velocity potential on the body, giving the hydrodynamic pressure, could be done with a finite difference scheme in this particular cases. However this scheme leads to numerical instabilities when considering freely moving bodies [Cointe(1989), Koo and Kim(2004)] with explicit time-stepping schemes. Moreover, since the hydrodynamic pressure was 
needed to solve the body motion equations, a method solving the mechanical and hydrodynamic problems simultaneously was required.

Four methods were proposed along the years in order to cope with this difficulty. The modal decomposition was first developed by Vinje and Brevig [Vinje and Brevig(1981)] and further implemented by Cointe [Cointe and Geyer(1990)]. A second method is the iterative method, used by Sen [Sen(1993)] and Cao [Cao et al.(1994)Cao, Beck, and Schultz, and based on a predictor-corrector loop to converge on the body accelerations. The indirect method was introduced by Wu and Eatock-Taylor Wu and Eatock Taylor(1996) and used by Kashiwagi [Kashiwagi(1996)]. It is the only method that does not require solving the Laplace equation for the time derivative of the velocity potential. However it solves directly the body motions without calculating the hydrodynamic force on the body. The last method was introduced simultaneously by Tanizawa Tanizawa(1995) and Van Daalen van Daalen(1993) and is called the Implicit Boundary method. Further details on these methods can be found in the previously mentioned references, but also in several reviews Tanizawa(2000), Koo and Kim(2004), Guerber(2011). Except for the indirect one, the three other methods require solving the Laplace equation for the time derivative of the velocity potential, the main difficulty lying in its body boundary condition.

Two expressions were given for this body boundary condition. One was proposed by Cointe [Cointe and Geyer(1990)], first in 2D and later extended by Van Daalen van Daalen(1993)] in 3D. The second was given by Tanizawa [Tanizawa(1995)] in 2D, and Berkvens [Berkvens(1998)] in 3D. Even if both expressions are implemented in several numerical flow solvers, their equivalence has not been proven yet. This paper will thus be dedicated to prove that these expression are equivalent. The four expressions were moreover developed using different notations and reference systems, that are not always explicited in the references. We thus provide here with a common ground for the comparison of those expressions.

The developments of both expressions are first provided, in 2D and 3D. The equivalence is then demonstrated, leading to a new unified expression, simplifying Cointe-van Daalen ones by suppressing second order derivatives with already known variables, and thus ensuring a more accurate estimation of the Body Boundary condition. A simple analytical case of a sphere in prescribed motion in an unbounded water domain is finally applied to ensure that the body condition is correct.

\section{THEORY}

\subsection{Potential flow theory}

Assuming a fluid to be incompressible and inviscid with irrotational flow, its flow velocity derives from a velocity potential $\phi$ which satisfies the Laplace Eq. [Newman(1977)]:

$$
\nabla^{2} \phi(x, y, z, t)=0
$$

in the fluid domain, $D$. The boundary of the fluid domain is $\partial D=\Gamma=\Gamma_{f s} \cup \Gamma_{b} \cup \Gamma_{w} \cup \Gamma_{d}$, see Fig 1 . 


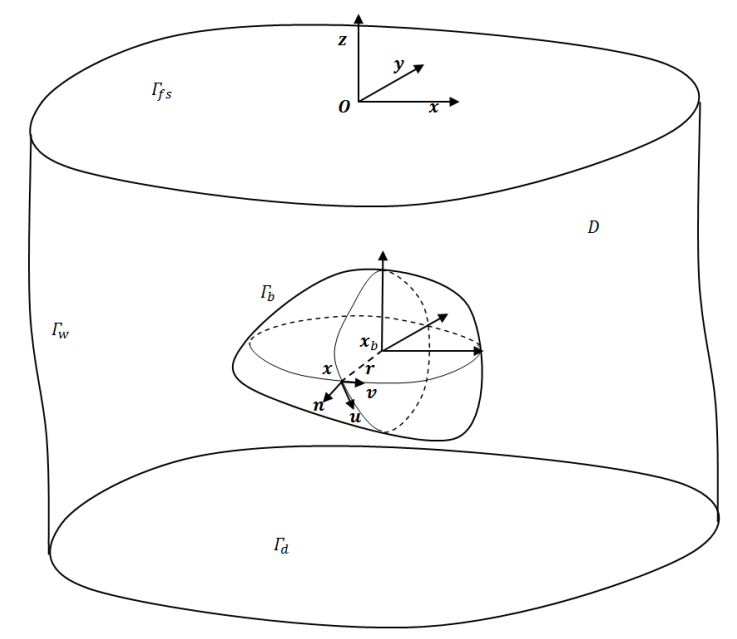

Figure 1: Domain definition : boundaries and reference frames

In the general case, without forward speed, it can be shown |Wehausen and Laitone(1960)| that the velocity potential is the solution of the following boundary value problem:

$$
\left\{\begin{array}{lr}
\nabla^{2} \phi=0 & \text { in the fluid domain } D \\
\frac{\partial \phi}{\partial t}=-g \eta-\frac{1}{2} \nabla \phi \cdot \nabla \phi & \text { on the free surface, } \Gamma_{f s} \\
\frac{\partial \eta}{\partial t}=\frac{\partial \phi}{\partial z}-\nabla \phi \cdot \nabla \eta & \text { on the free surface, } \Gamma_{f s} \\
\frac{\partial \phi}{\partial n}=\mathbf{V}_{\mathbf{b}} \cdot \mathbf{n} & \text { on the body, } \Gamma_{b} \\
\frac{\partial \phi}{\partial n}=0 & \text { on boundaries far from the body, } \Gamma_{w}
\end{array}\right.
$$

The free surface elevation is denoted by the single-valued variable $\eta . g$ is the gravitational constant, $\mathbf{V}_{b}$ the body velocity and $\mathbf{n}$ the normal vector pointing outsides from the fluid.

Using Green's Second Identity, it can be shown that the resolution of the 3D Laplace equation in the fluid domain can be reduced to a Boundary Value Problem (BVP) for the velocity potential. For any point $\mathbf{x} \in D$,

$$
\alpha(\mathbf{x}) \phi(\mathbf{x})=\int_{\Gamma}\left[\frac{\partial \phi}{\partial n}\left(\mathbf{x}_{l}\right) G\left(\mathbf{x}, \mathbf{x}_{l}\right)-\phi\left(\mathbf{x}_{l}\right) \frac{\partial G}{\partial n}\left(\mathbf{x}, \mathbf{x}_{l}\right)\right] d \Gamma
$$

where $\alpha(\mathbf{x})=\iint_{\Gamma} \frac{\partial G\left(\mathbf{x}, \mathbf{x}_{l}\right)}{\partial n} d \Gamma$ is the solid angle from which the closed surface $\Gamma$ is seen from point $\mathbf{x} \in \partial \Gamma$ and $G\left(\mathbf{x}, \mathbf{x}_{l}\right)$ is a Green function.

Solving the velocity potential BVP gives access to its normal derivative on the freesurface and the velocity potential on the body. While the first is used to compute the 
free-surface equations, solved in a Lagrangian way to get the propagation of waves on the free-surface, the latter is used to calculate the pressure force on the body. The body motions can then be computed, according to the equations of motion.

\subsection{Equations of motion}

The position, velocity and acceleration, $(\mathbf{x}, \dot{\mathbf{x}}, \ddot{\mathbf{x}})$, of a point on the body can be expressed in the global reference frame using those of the center of gravity, $\left(\mathbf{x}_{b}, \dot{\mathbf{x}}_{b}, \ddot{\mathbf{x}}_{b}\right)$, and its local coordinates, $\mathbf{r}$.

$$
\left\{\begin{array}{l}
\mathbf{x}=\mathbf{x}_{b}+\mathbf{r} \\
\dot{\mathbf{x}}=\dot{\mathbf{x}}_{b}+\dot{\theta} \wedge \mathbf{r} \\
\ddot{\mathbf{x}}=\ddot{\mathbf{x}}_{b}+\ddot{\theta} \wedge \mathbf{r}+\dot{\theta} \wedge \dot{\theta} \wedge \mathbf{r}
\end{array}\right.
$$

where $\left(\dot{\mathbf{x}}_{b}, \ddot{\mathbf{x}}_{b}\right)$ are the velocity and acceleration of the body center of gravity, and $\theta$ is the body rotational vector. In the inertial reference frame following the body motions, $\mathbf{r}$ is constant.

The body motion equations in translation are obtained according to Newton's law:

$$
\mathbf{M} \cdot \ddot{\mathbf{x}}_{b}=\mathbf{F}_{H}+\mathbf{M} \cdot \mathbf{g}+\sum \mathbf{F}_{e x t}
$$

where $\mathbf{M}$ is the mass matrix, $\ddot{\mathbf{x}}_{b}$ is the acceleration in translation of the center of gravity of the body in the global reference frame, $\mathbf{F}_{H}$ and $\mathbf{F}_{e x t}$ are respectively the hydrodynamic and external forces. The latter represents any forces applied on the body by external systems, ranging from Power Take-Off (PTO) for energy converters, mooring, to viscous forces which can not be modeled with the potential flow theory.

\subsection{Hydrodynamic Force}

The hydrodynamic force and moment are obtained by integrating the pressure on the wet body surface (normal vector pointing outsides from the fluid).

$$
\mathbf{F}_{H}=-\iint_{\Gamma_{b}} p(\mathbf{x}) \mathbf{n}(\mathbf{x}) d \Gamma
$$

The pressure on the body is given by Bernoulli equation, with the free surface pressure being equal to the atmospheric pressure:

$$
p=-\rho\left(\frac{\partial \phi}{\partial t}+\frac{1}{2} \nabla \phi \cdot \nabla \phi+g z\right)
$$

The velocity potential is known on the body, as the solution of the BVP. Its spatial derivatives can be computed numerically using local approximation of the potential, or any particular discretization method. The time derivative of the velocity potential is however a priori not known on the body and needs to be determined. 


\subsection{Velocity Potential Time Derivative}

For a body undergoing prescribed motion, the equations of motion, and thus the hydrodynamic loads, do not need to be computed during the simulation. Since the velocity potential is known on the body at each time-step, a finite difference scheme can be used in post-processing.

However, for a body undergoing free motion, the finite difference scheme used with an explicit time-stepping formulation, can lead to instabilities Cointe(1989), Koo and Kim(2004). Solving a BVP written for the time derivative of the velocity potential is an alternate solution.

$$
\triangle \phi=0 \Longrightarrow \triangle \frac{\partial \phi}{\partial t}=0, \text { in } D
$$

This BVP is similar to the one for the velocity potential. The boundary conditions are mixed: Neumann for the material boundaries (body, seabed and numerical walls) and Dirichlet for the free-surface. The free-surface boundary condition is given by the dynamic free-surface condition, which can be written in its most general form as

$$
\frac{\partial \phi}{\partial t}=g \eta+\frac{1}{2} \nabla \phi \cdot \nabla \phi, \text { on } z=\eta(x, y, t)
$$

The boundary condition on the seabed, $\Gamma_{d}$, and outer boundaries, $\Gamma_{w}$ can simply be written as

$$
\frac{\partial^{2} \phi}{\partial n \partial t}=0
$$

since these boundaries are fixed in time.

The main difficulty lies in the treatment of the body boundary condition, associated to the time evolving body. This is detailed in the next section.

\section{EXPRESSIONS FOR THE BODY BOUNDARY CON- DITION}

Two expressions have been given, by Cointe [Cointe and Geyer(1990) and Tanizawa [Tanizawa(1995)] for this particular boundary condition. In order to demonstrate the equivalence of both expressions, their development are recalled first, in 2D and 3D. The proof of their equivalence is then shown, leading to a new unified expression.

The main difference between the two expressions is located in the acceleration used to express the body boundary condition. Cointe used the acceleration of the point considered on the body, denoted $\ddot{\mathbf{x}}$, while Tanizawa used the acceleration of a fluid particle sliding on the body boundary, denoted $\mathbf{a}$. It is however possible to express one as a function of the other, knowing the body velocity and the velocity potential on the body. 


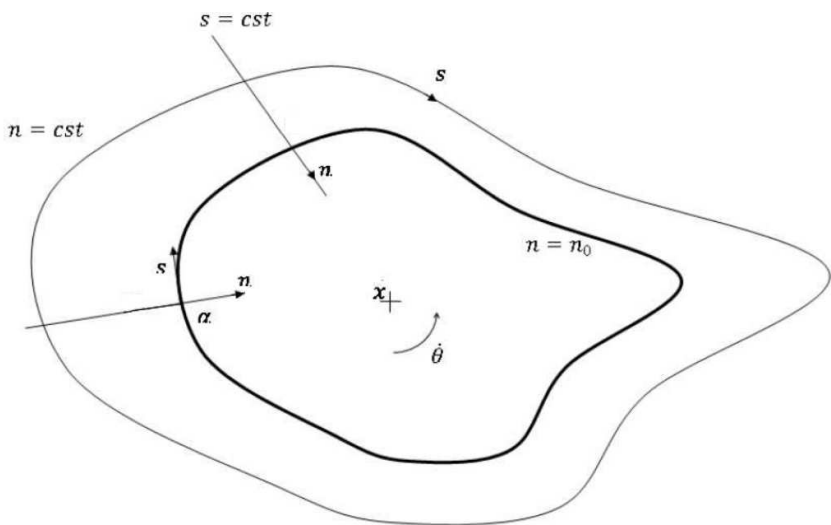

(a) 2D Cointe basis

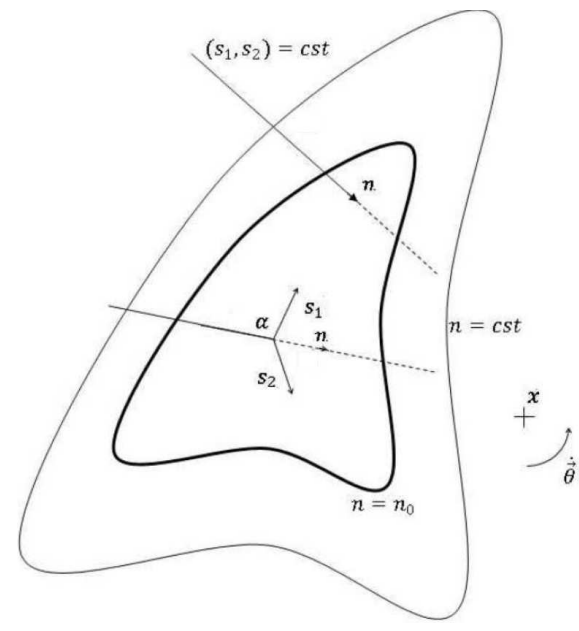

(b) 3D van Daalen basis

Figure 2: bases used by Cointe and van Daalen

\subsection{Cointe expression}

Cointe Cointe(1989) expressed the body boundary condition for the time derivative of the velocity potential in terms of the time derivative of the body condition for the velocity potential. For a given point $\mathbf{x}$ of the body, with acceleration $\ddot{\mathbf{x}}$, Cointe gave the following $2 \mathrm{D}$ expression for the body condition:

$$
\frac{\partial^{2} \phi}{\partial n \partial t}=(\ddot{\mathbf{x}} \cdot \mathbf{n})-\dot{\theta}\left(\frac{\partial \phi}{\partial s}-(\dot{\mathbf{x}} \cdot \mathbf{s})\right)-(\dot{\mathbf{x}} \cdot \mathbf{s})\left(\frac{\partial^{2} \phi}{\partial n \partial s}+\frac{1}{R} \frac{\partial \phi}{\partial s}\right)+(\dot{\mathbf{x}} \cdot \mathbf{n})\left(\frac{\partial^{2} \phi}{\partial s^{2}}-\frac{1}{R} \frac{\partial \phi}{\partial n}\right)
$$

where $\dot{\theta}$ is the rotational velocity in the global coordinate system, $\mathbf{s}$ is the local tangent vector, $\mathbf{n}$ is the normal vector at this point and $k=\frac{1}{R}$ is the local curvature on the body. It is to be noted that Cointe used an indirect local basis $(\mathbf{s}, \mathbf{n})$ with a normal vector pointing inside the body (see Fig. 2a).

Van Daalen van Daalen(1993) then extended this expression to 3D:

$$
\begin{aligned}
\frac{\partial^{2} \phi}{\partial n \partial t}=(\ddot{\mathbf{x}} \cdot \mathbf{n}) & +\left(\dot{\theta} \cdot \mathbf{s}_{2}\right)\left(\left(\dot{\mathbf{x}} \cdot \mathbf{s}_{1}\right)-\frac{\partial \phi}{\partial s_{1}}\right)-\left(\dot{\theta} \cdot \mathbf{s}_{1}\right)\left(\left(\dot{\mathbf{x}} \cdot \mathbf{s}_{2}\right)-\frac{\partial \phi}{\partial s_{2}}\right) \\
& -\left(\dot{\mathbf{x}} \cdot \mathbf{s}_{1}\right)\left(\frac{1}{R_{1}} \frac{\partial \phi}{\partial s_{1}}+\frac{\partial^{2} \phi}{\partial s_{1} \partial n}\right)-\left(\dot{\mathbf{x}} \cdot \mathbf{s}_{2}\right)\left(\frac{1}{R_{2}} \frac{\partial \phi}{\partial s_{2}}+\frac{\partial^{2} \phi}{\partial s_{2} \partial n}\right) \\
& +(\dot{\mathbf{x}} \cdot \mathbf{n})\left(\frac{\partial^{2} \phi}{\partial s_{1}^{2}}+\frac{\partial^{2} \phi}{\partial s_{2}^{2}}-\left(\frac{1}{R_{1}}+\frac{1}{R_{2}}\right) \frac{\partial \phi}{\partial n}\right)
\end{aligned}
$$

where $\left(\mathbf{s}_{1}, \mathbf{s}_{2}\right)$ are the two local tangent vectors and $k_{1}=\frac{1}{R_{1}}, k_{2}=\frac{1}{R_{2}}$ are the local curvatures associated to $\left(\mathbf{s}_{1}, \mathbf{s}_{2}\right)$. Van Daalen used a direct local basis, with a normal vector pointing also inside the body(see Fig. 2b) . 


\subsubsection{D Expression}

For a body with a direct local curvilinear system $(O, s, n)$ defined from the tangential and normal vectors $(\mathbf{s}, \mathbf{n})$, the curves $s=$ constant are straight lines corresponding to the normal vectors. The curves $n=$ constant are homothetic contours of the body surface, with $n=n_{0}$ corresponding to the body surface. The normal vector is now chosen pointing outside the body, see Fig. 3 ,

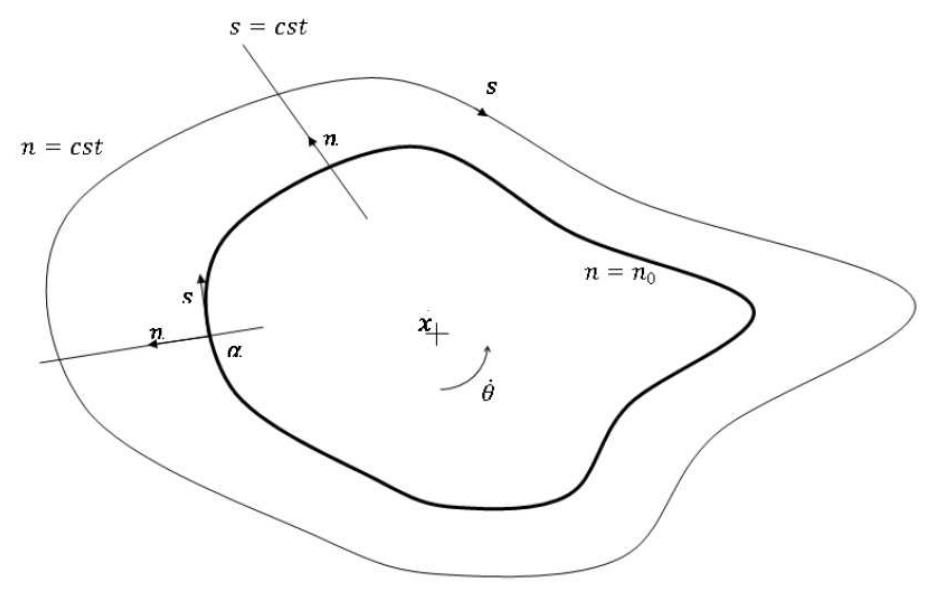

Figure 3: Local curvilinear system

In Cartesian coordinates, the position of a point is written as $\mathbf{r}=x \cdot \mathbf{e}_{x}+y \cdot \mathbf{e}_{y}$. In the curvilinear system, the local vectors are defined as:

$$
\mathbf{n}=\frac{1}{h_{n}} \frac{\partial \mathbf{r}}{\partial n} \quad \text { and } \quad \mathbf{s}=\frac{1}{h_{s}} \frac{\partial \mathbf{r}}{\partial s}
$$

The scale factors are defined as:

$$
h_{n}=|\mathbf{n}| \quad \text { and } \quad h_{s}=|\mathbf{s}|
$$

According to the definition of the curvilinear system $(s, n)$, the normal scale vector variations can be expressed, for all space variables $(s, n)$ and time variable $t$, as:

$$
h_{n}=1, \quad \frac{\partial h_{n}}{\partial s}=0, \quad \frac{\partial h_{n}}{\partial n}=0 \text { and } \quad \frac{\partial h_{n}}{\partial t}=0
$$

The tangent vector $\mathbf{s}$ is also a unit vector on the contour $\left(n=n_{0}\right)$ for all $s$. However, its variation along $n$ is non-zero, and can be expressed in terms of the local curvature radius $R$ :

$$
h_{s}=1, \quad \frac{\partial h_{s}}{\partial n}=\frac{h_{s}}{R} \quad \text { and } \quad \frac{\partial h_{s}}{\partial s}=0
$$

In curvilinear coordinates, the derivative of any unit vector $\mathbf{h}_{i}$ along the local parameter $w_{j}$ is defined as:

$$
\frac{\partial \mathbf{h}_{i}}{\partial w_{j}}=\frac{1}{h_{i}} \frac{\partial h_{j}}{\partial w_{i}} \mathbf{h}_{j}-\delta_{i j} \sum_{k, k \neq i} \frac{1}{h_{k}} \frac{\partial h_{i}}{\partial w_{k}} \mathbf{h}_{k}
$$


In our case, the local derivatives of the unit vectors are then:

$$
\left\{\begin{array}{l}
\frac{\partial \mathbf{s}}{\partial s}=\frac{1}{h_{s}} \frac{\partial h_{s}}{\partial s} \mathbf{s}-\frac{1}{h_{n}} \frac{\partial h_{s}}{\partial n} \mathbf{n}=-\frac{\partial h_{s}}{\partial n} \mathbf{n} \\
\frac{\partial \mathbf{s}}{\partial n}=\frac{1}{h_{s}} \frac{\partial h_{n}}{\partial s} \mathbf{n}=\mathbf{0} \\
\frac{\partial \mathbf{n}}{\partial s}=\frac{1}{h_{n}} \frac{\partial h_{s}}{\partial n} \mathbf{s}=\frac{\partial h_{s}}{\partial n} \mathbf{s} \\
\frac{\partial \mathbf{n}}{\partial n}=\frac{1}{h_{n}} \frac{\partial h_{n}}{\partial n} \mathbf{n}-\frac{1}{h_{s}} \frac{\partial h_{n}}{\partial s} \mathbf{s}=\mathbf{0}
\end{array}\right.
$$

In curvilinear coordinates, the derivative operators can be expressed as:

$$
\left\{\begin{aligned}
\nabla \phi & =\frac{1}{h_{i}} \frac{\partial \phi}{\partial w_{i}} h^{i}=\frac{1}{h_{s}} \frac{\partial \phi}{\partial s} \cdot \mathbf{s}+\frac{\partial \phi}{\partial n} \cdot \mathbf{n} \\
\triangle \phi & =\nabla \cdot(\nabla \phi)=\frac{1}{h_{s}^{2}} \frac{\partial^{2} \phi}{\partial s^{2}}+\frac{1}{h_{s}} \frac{\partial h_{s}}{\partial n} \frac{\partial \phi}{\partial n}+\frac{\partial^{2} \phi}{\partial n^{2}}=0
\end{aligned}\right.
$$

The time derivative of the velocity potential, when following the body motion, is defined by $\frac{\delta \phi}{\delta t}$. This derivative is a material derivative associated to the velocity of the body point, $\dot{\mathbf{x}}$. It can thus be expressed as

$$
\frac{\delta \phi}{\delta t}=\frac{\partial \phi}{\partial t}+\dot{\mathbf{x}} \cdot \nabla \phi=\frac{\partial \phi}{\partial t}+(\dot{\mathbf{x}} \cdot \mathbf{s}) \frac{1}{h_{s}} \frac{\partial \phi}{\partial s}+(\dot{\mathbf{x}} \cdot \mathbf{n}) \frac{\partial \phi}{\partial n}
$$

The local unit vectors follow the body motions; their material time derivatives can thus be written as:

$$
\frac{\delta \mathbf{s}}{\delta t}=\dot{\theta} \mathbf{n} \quad \text { and } \quad \frac{\delta \mathbf{n}}{\delta t}=-\dot{\theta} \mathbf{s}
$$

and their partial time derivatives are then:

$$
\left\{\begin{array}{l}
\frac{\partial \mathbf{s}}{\partial t}=\frac{\delta \mathbf{s}}{\partial t}-(\dot{\mathbf{x}} \cdot \mathbf{s}) \frac{1}{h_{s}} \frac{\partial \mathbf{s}}{\partial s}-(\dot{\mathbf{x}} \cdot \mathbf{n}) \frac{\partial \mathbf{s}}{\partial n}=\dot{\theta} \cdot \mathbf{n}+(\dot{\mathbf{x}} \cdot \mathbf{s}) \frac{1}{h_{s}} \frac{\partial h_{s}}{\partial n} \cdot \mathbf{n} \\
\frac{\partial \mathbf{n}}{\partial t}=\frac{\delta \mathbf{n}}{\partial t}-(\dot{\mathbf{x}} \cdot \mathbf{s}) \frac{1}{h_{s}} \frac{\partial \mathbf{n}}{\partial s}-(\dot{\mathbf{x}} \cdot \mathbf{n}) \frac{\partial \mathbf{n}}{\partial n}=-\dot{\theta} \cdot \mathbf{s}-(\dot{\mathbf{x}} \cdot \mathbf{s}) \frac{1}{h_{s}} \frac{\partial h_{s}}{\partial n} \cdot \mathbf{s}
\end{array}\right.
$$

The acceleration of a point $\mathbf{x}$ of the body can be written, using the material derivative, as:

$$
\begin{aligned}
\ddot{\mathbf{x}} & =\frac{\delta \dot{\mathbf{x}}}{\delta t}=\frac{\delta}{\delta t}((\dot{\mathbf{x}} \cdot \mathbf{s}) \mathbf{s}+(\dot{\mathbf{x}} \cdot \mathbf{n}) \mathbf{n}) \\
& =\left(\frac{\delta}{\delta t}(\dot{\mathbf{x}} \cdot \mathbf{s})-\dot{\theta}(\dot{\mathbf{x}} \cdot \mathbf{n})\right) \mathbf{s}+\left(\frac{\delta}{\delta t}(\dot{\mathbf{x}} \cdot \mathbf{n})+\dot{\theta}(\dot{\mathbf{x}} \cdot \mathbf{s})\right) \mathbf{n}
\end{aligned}
$$

from which it follows that:

$$
\ddot{\mathbf{x}} \cdot \mathbf{n}=\frac{\delta}{\delta t}(\dot{\mathbf{x}} \cdot \mathbf{n})+\dot{\theta}(\dot{\mathbf{x}} \cdot \mathbf{s})
$$


The body boundary condition gives $\dot{\mathbf{x}} \cdot \mathbf{n}=\nabla \phi \cdot \mathbf{n}$. Using this, Eq.(24) becomes:

$$
\begin{aligned}
\ddot{\mathbf{x}} \cdot \mathbf{n} & =\frac{\delta}{\delta t}(\nabla \phi \cdot \mathbf{n})+\dot{\theta}(\dot{\mathbf{x}} \cdot \mathbf{s})=\frac{\delta}{\delta t}(\nabla \phi) \cdot \mathbf{n}+\nabla \phi \cdot \frac{\delta \mathbf{n}}{\delta t}+\dot{\theta}(\dot{\mathbf{x}} \cdot \mathbf{s}) \\
& =\frac{\partial^{2} \phi}{\partial n \partial t}+((\dot{\mathbf{x}} \cdot \nabla) \nabla \phi) \cdot \mathbf{n}+\dot{\theta}\left((\dot{\mathbf{x}} \cdot \mathbf{s})-\frac{1}{h_{s}} \frac{\partial \phi}{\partial s}\right)
\end{aligned}
$$

Using the derivations established in Eq. (19) to express the local derivatives in curvilinear coordinates, the advection term can be expressed as:

$$
(\dot{\mathbf{x}} \cdot \nabla) \nabla \phi=\frac{(\dot{\mathbf{x}} \cdot \mathbf{s})}{h_{s}} \frac{\partial}{\partial s}\left(\frac{1}{h_{s}} \frac{\partial \phi}{\partial s} \mathbf{s}+\frac{1}{h_{n}} \frac{\partial \phi}{\partial n} \mathbf{n}\right)+(\dot{\mathbf{x}} \cdot \mathbf{n}) \frac{1}{h_{n}} \frac{\partial}{\partial n}\left(\frac{1}{h_{s}} \frac{\partial \phi}{\partial s} \mathbf{s}+\frac{1}{h_{n}} \frac{\partial \phi}{\partial n} \mathbf{n}\right)
$$

Those of Eq. (18) yields

$$
\begin{aligned}
((\dot{\mathbf{x}} \cdot \nabla) \nabla \phi) \cdot \mathbf{n} & =\frac{(\dot{\mathbf{x}} \cdot \mathbf{s})}{h_{s}}\left(-\frac{1}{h_{s}} \frac{\partial h_{s}}{\partial n} \frac{\partial \phi}{\partial s}+\frac{1}{h_{n}} \frac{\partial^{2} \phi}{\partial s \partial n}\right)+(\dot{\mathbf{x}} \cdot \mathbf{n}) \frac{1}{h_{n}^{2}} \frac{\partial^{2} \phi}{\partial n^{2}} \\
& =\frac{(\dot{\mathbf{x}} \cdot \mathbf{s})}{h_{s}}\left(-\frac{1}{h_{s}} \frac{\partial h_{s}}{\partial n} \frac{\partial \phi}{\partial s}+\frac{1}{h_{n}} \frac{\partial^{2} \phi}{\partial s \partial n}\right)-(\dot{\mathbf{x}} \cdot \mathbf{n})\left(\frac{1}{h_{s}^{2}} \frac{\partial^{2} \phi}{\partial s^{2}}+\frac{1}{h_{s}} \frac{\partial h_{s}}{\partial n} \frac{\partial \phi}{\partial n}\right)
\end{aligned}
$$

The Laplace equation has been used to get rid of the second normal derivative of the potential.

$$
\frac{1}{h_{n}^{2}} \frac{\partial^{2} \phi}{\partial n^{2}}=-\frac{1}{h_{s}^{2}} \frac{\partial^{2} \phi}{\partial s^{2}}-\frac{1}{h_{s}} \frac{\partial h_{s}}{\partial n} \frac{\partial \phi}{\partial n}
$$

Eq.(25) then becomes:

$$
\begin{aligned}
\ddot{\mathbf{x}} \cdot \mathbf{n}= & \frac{\partial^{2} \phi}{\partial n \partial t}+\dot{\theta}\left((\dot{\mathbf{x}} \cdot \mathbf{s})-\frac{1}{h_{s}} \frac{\partial \phi}{\partial s}\right)+\frac{(\dot{\mathbf{x}} \cdot \mathbf{s})}{h_{s}}\left(-\frac{1}{h_{s}} \frac{\partial h_{s}}{\partial n} \frac{\partial \phi}{\partial s}+\frac{1}{h_{n}} \frac{\partial^{2} \phi}{\partial s \partial n}\right) \\
& -(\dot{\mathbf{x}} \cdot \mathbf{n})\left(\frac{1}{h_{s}^{2}} \frac{\partial^{2} \phi}{\partial s^{2}}+\frac{1}{h_{s}} \frac{\partial h_{s}}{\partial n} \frac{\partial \phi}{\partial n}\right)
\end{aligned}
$$

leading to:

$$
\begin{aligned}
\frac{\partial^{2} \phi}{\partial n \partial t}= & \ddot{\mathbf{x}} \cdot \mathbf{n}+\dot{\theta}\left(\frac{1}{h_{s}} \frac{\partial \phi}{\partial s}-(\dot{\mathbf{x}} \cdot \mathbf{s})\right)+(\dot{\mathbf{x}} \cdot \mathbf{s})\left(\frac{1}{h_{s}^{2}} \frac{\partial h_{s}}{\partial n} \frac{\partial \phi}{\partial s}-\frac{1}{h_{s} h_{n}} \frac{\partial^{2} \phi}{\partial s \partial n}\right) \\
& +(\dot{\mathbf{x}} \cdot \mathbf{n})\left(\frac{1}{h_{s}^{2}} \frac{\partial^{2} \phi}{\partial s^{2}}+\frac{1}{h_{s}} \frac{\partial h_{s}}{\partial n} \frac{\partial \phi}{\partial n}\right)
\end{aligned}
$$

Replacing the scale factors $h_{s}=1=h_{n}$ and their variation $\frac{1}{h_{s}} \frac{\partial h_{s}}{\partial n}=\frac{1}{R}$ by their respective values, the above equation becomes:

$$
\frac{\partial^{2} \phi}{\partial n \partial t}=\ddot{\mathbf{x}} \cdot \mathbf{n}+\dot{\theta}\left(\frac{\partial \phi}{\partial s}-(\dot{\mathbf{x}} \cdot \mathbf{s})\right)+(\dot{\mathbf{x}} \cdot \mathbf{s})\left(\frac{1}{R} \frac{\partial \phi}{\partial s}-\frac{\partial^{2} \phi}{\partial s \partial n}\right)+(\dot{\mathbf{x}} \cdot \mathbf{n})\left(\frac{\partial^{2} \phi}{\partial s^{2}}+\frac{1}{R} \frac{\partial \phi}{\partial n}\right)
$$




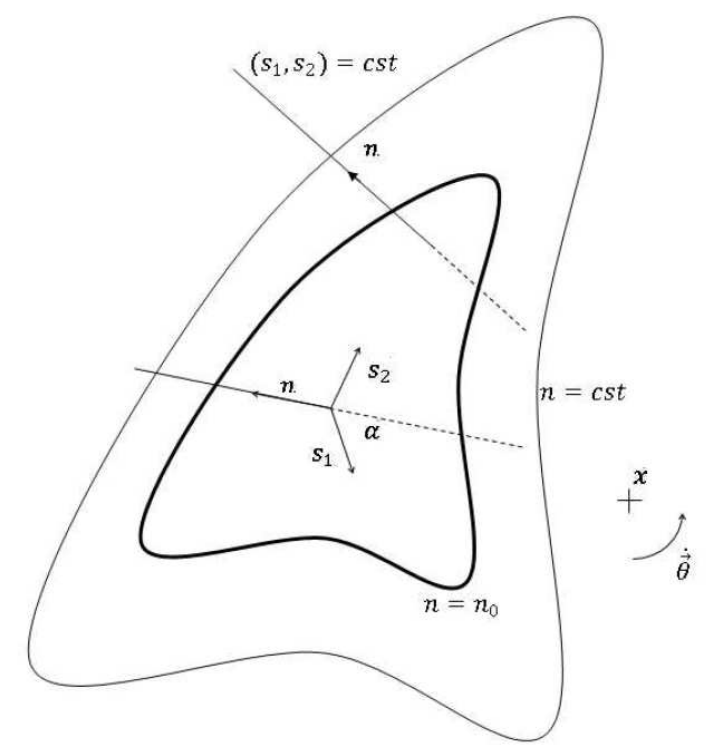

Figure 4: 3D curvilinear basis

To compare this with the Cointe expression for an indirect local basis, let's consider an opposite tangential vector $\left(\mathbf{s}^{\prime}=-\mathbf{s}\right)$

$$
\frac{\partial}{\partial s^{\prime}}=-\frac{\partial}{\partial s}, \quad \dot{\mathbf{x}} \cdot \mathbf{s}^{\prime}=-\dot{\mathbf{x}} \cdot \mathbf{s} \quad \text { and } \quad \ddot{\mathbf{x}} \cdot \mathbf{s}^{\prime}=-\ddot{\mathbf{x}} \cdot \mathbf{s}
$$

and, since the normal vector is pointing in an opposite direction, we also have to consider an opposite curvature radius: $R^{\prime}=-R$. The Cointe expression, Eq.(11), is then recovered:

$$
\frac{\partial^{2} \phi}{\partial n \partial t}=\ddot{\mathbf{x}} \cdot \mathbf{n}-\dot{\theta}\left(\frac{\partial \phi}{\partial s^{\prime}}-\left(\dot{\mathbf{x}} \cdot \mathbf{s}^{\prime}\right)\right)-\left(\dot{\mathbf{x}} \cdot \mathbf{s}^{\prime}\right)\left(\frac{\partial^{2} \phi}{\partial s^{\prime} \partial n}+\frac{1}{R^{\prime}} \frac{\partial \phi}{\partial s^{\prime}}\right)+(\dot{\mathbf{x}} \cdot \mathbf{n})\left(\frac{\partial^{2} \phi}{\partial s^{\prime 2}}-\frac{1}{R^{\prime}} \frac{\partial \phi}{\partial n}\right)
$$

\subsubsection{D Expression}

A similar 3D direct curvilinear basis is introduced, with a normal vector pointing outside the body, see Fig. 4 . The unit vector derivatives can be written in a general form as:

$$
\frac{\partial \mathbf{h}_{i}}{\partial w_{j}}=\frac{1}{h_{i}} \frac{\partial h_{j}}{\partial w_{i}} \mathbf{h}_{j}-\delta_{i j} \sum_{k \neq i} \frac{1}{h_{k}} \frac{\partial h_{i}}{\partial w_{k}} \mathbf{h}_{k}
$$


leading in our case to:

$$
\left\{\begin{array}{l}
\frac{\partial \mathbf{s}_{1}}{\partial s_{1}}=-\frac{1}{h_{s_{2}}} \frac{\partial h_{s_{1}}}{\partial s_{2}} \mathbf{s}_{2}-\frac{1}{h_{n}} \frac{\partial h_{s_{1}}}{\partial n} \mathbf{n} \\
\frac{\partial \mathbf{s}_{1}}{\partial s_{2}}=\frac{1}{h_{s_{1}}} \frac{\partial h_{s_{2}}}{\partial s_{1}} \mathbf{s}_{2} \\
\frac{\partial \mathbf{s}_{1}}{\partial n}=\frac{1}{h_{s_{1}}} \frac{\partial h_{n}}{\partial s_{1}} \mathbf{n} \\
\frac{\partial \mathbf{s}_{2}}{\partial s_{1}}=\frac{1}{h_{s_{2}}} \frac{\partial h_{s_{1}}}{\partial s_{2}} \mathbf{s}_{1} \\
\frac{\partial \mathbf{s}_{2}}{\partial s_{2}}=-\frac{1}{h_{s_{1}}} \frac{\partial h_{s_{2}}}{\partial s_{1}} \mathbf{s}_{1}-\frac{1}{h_{n}} \frac{\partial h_{s_{2}}}{\partial n} \mathbf{n} \\
\frac{\partial \mathbf{s}_{2}}{\partial n}=\frac{1}{h_{s_{2}}} \frac{\partial h_{n}}{\partial s_{2}} \mathbf{n} \\
\frac{\partial \mathbf{n}}{\partial s_{1}}=\frac{1}{h_{n}} \frac{\partial h_{s_{1}}}{\partial n} \mathbf{s}_{\mathbf{1}} \\
\frac{\partial \mathbf{n}}{\partial s_{2}}=\frac{1}{h_{n}} \frac{\partial h_{s_{2}}}{\partial n} \mathbf{s}_{\mathbf{2}} \\
\frac{\partial \mathbf{n}}{\partial n}=-\frac{1}{h_{s_{1}}} \frac{\partial h_{n}}{\partial s_{1}} \mathbf{s}_{1}-\frac{1}{h_{s_{2}}} \frac{\partial h_{n}}{\partial s_{2}} \mathbf{s}_{2}
\end{array}\right.
$$

where the scale factor $h_{k}$ is defined by:

$$
h_{k}^{2}=\sum_{m=1}^{3} \frac{\partial x_{m}^{2}}{\partial s_{k}}
$$

Thus, for any $\mathbf{x}$ strictly on the body surface, $\mathbf{x}=\mathbf{x}\left(s_{1}, s_{2}\right)$, which means that $h_{n}$ is constant on the body surface, i.e.:

$$
\left\{\begin{array}{l}
\frac{\partial h_{n}}{\partial s_{1}}=0 \\
\frac{\partial h_{n}}{\partial s_{2}}=0
\end{array}\right.
$$

The tangential scale factors $h_{s_{1}}$ and $h_{s_{2}}$ are also constant on the body surface, however their normal derivative is given by the local curvature:

$$
\left\{\begin{array}{l}
\gamma_{1}=\frac{1}{R_{1}}=\frac{1}{h_{s_{1}}} \frac{\partial h_{s_{1}}}{\partial n} \\
\gamma_{2}=\frac{1}{R_{2}}=\frac{1}{h_{s_{2}}} \frac{\partial h_{s_{2}}}{\partial n}
\end{array}\right.
$$


The derivative operators can then be written as:

$$
\left\{\begin{aligned}
\nabla \phi & =\frac{1}{h_{s_{1}}} \frac{\partial \phi}{\partial s_{1}} \mathbf{s}_{1}+\frac{1}{h_{s_{2}}} \frac{\partial \phi}{\partial s_{2}} \mathbf{s}_{2}+\frac{1}{h_{n}} \frac{\partial \phi}{\partial n} \mathbf{n} \\
\triangle \phi & =\frac{1}{h_{s_{1}}^{2}} \frac{\partial^{2} \phi}{\partial s_{1}^{2}}+\frac{1}{h_{s_{2}}^{2}} \frac{\partial^{2} \phi}{\partial s_{2}^{2}}+\frac{1}{h_{n}^{2}} \frac{\partial^{2} \phi}{\partial n^{2}}+\frac{1}{h_{n}^{2}}\left(\frac{1}{h_{s_{1}}} \frac{\partial h_{s_{1}}}{\partial n}+\frac{1}{h_{s_{2}}} \frac{\partial h_{s_{2}}}{\partial n}\right) \frac{\partial \phi}{\partial n}
\end{aligned}\right.
$$

and the time derivatives are:

$$
\left\{\begin{array}{l}
\frac{\delta \mathbf{s}_{1}}{\delta t}=\dot{\theta} \wedge \mathbf{s}_{1}=(\dot{\theta} \cdot \mathbf{n}) \mathbf{s}_{2}-\left(\dot{\theta} \cdot \mathbf{s}_{2}\right) \mathbf{n} \\
\frac{\delta \mathbf{s}_{2}}{\delta t}=\dot{\theta} \wedge \mathbf{s}_{2}=\left(\dot{\theta} \cdot \mathbf{s}_{1}\right) \mathbf{n}-(\dot{\theta} \cdot \mathbf{n}) \mathbf{s}_{1} \\
\frac{\delta \mathbf{n}}{\delta t}=\dot{\theta} \wedge \mathbf{n}=\left(\dot{\theta} \cdot \mathbf{s}_{2}\right) \mathbf{s}_{1}-\left(\dot{\theta} \cdot \mathbf{s}_{1}\right) \mathbf{s}_{2} \\
\frac{\delta}{\delta t}=\frac{\partial}{\partial t}+(\dot{\mathbf{x}} \cdot \nabla)
\end{array}\right.
$$

The acceleration of a point on the body surface is written using the material derivative, in the same way as for the $2 \mathrm{D}$ expression.:

$$
\begin{aligned}
\ddot{\mathbf{x}}= & \frac{\delta \dot{\mathbf{x}}}{\delta t}=\frac{\delta}{\delta t}\left(\left(\dot{\mathbf{x}} \cdot \mathbf{s}_{1}\right) \mathbf{s}_{1}+\left(\dot{\mathbf{x}} \cdot \mathbf{s}_{2}\right) \mathbf{s}_{2}+(\dot{\mathbf{x}} \cdot \mathbf{n}) \mathbf{n}\right) \\
= & \frac{\delta}{\delta t}\left(\dot{\mathbf{x}} \cdot \mathbf{s}_{1}\right) \mathbf{s}_{1}+\left(\dot{\mathbf{x}} \cdot \mathbf{s}_{1}\right)\left((\dot{\theta} \cdot \mathbf{n}) \mathbf{s}_{2}-\left(\dot{\theta} \cdot \mathbf{s}_{2}\right) \mathbf{n}\right) \\
& +\frac{\delta}{\delta t}\left(\dot{\mathbf{x}} \cdot \mathbf{s}_{2}\right) \mathbf{s}_{2}+\left(\dot{\mathbf{x}} \cdot \mathbf{s}_{2}\right)\left(\left(\dot{\theta} \cdot \mathbf{s}_{1}\right) \mathbf{n}-(\dot{\theta} \cdot \mathbf{n}) \mathbf{s}_{1}\right) \\
& +\frac{\delta}{\delta t}(\dot{\mathbf{x}} \cdot \mathbf{n}) \mathbf{n}+(\dot{\mathbf{x}} \cdot \mathbf{n})\left(\left(\dot{\theta} \cdot \mathbf{s}_{2}\right) \mathbf{s}_{1}-\left(\dot{\theta} \cdot \mathbf{s}_{1}\right) \mathbf{s}_{2}\right)
\end{aligned}
$$

It follows that, using the body boundary condition:

$$
\begin{aligned}
\ddot{\mathbf{x}} \cdot \mathbf{n}= & \frac{\delta}{\delta t}(\dot{\mathbf{x}} \cdot \mathbf{n})+\left(\dot{\mathbf{x}} \cdot \mathbf{s}_{2}\right)\left(\dot{\theta} \cdot \mathbf{s}_{1}\right)-\left(\dot{\mathbf{x}} \cdot \mathbf{s}_{1}\right)\left(\dot{\theta} \cdot \mathbf{s}_{2}\right) \\
= & \frac{\delta}{\delta t}(\nabla \phi \cdot \mathbf{n})+\left(\dot{\mathbf{x}} \cdot \mathbf{s}_{2}\right)\left(\dot{\theta} \cdot \mathbf{s}_{1}\right)-\left(\dot{\mathbf{x}} \cdot \mathbf{s}_{1}\right)\left(\dot{\theta} \cdot \mathbf{s}_{2}\right) \\
= & \frac{\delta \nabla \phi}{\delta t} \cdot \mathbf{n}+\nabla \phi \frac{\delta \mathbf{n}}{\delta t}+\left(\dot{\mathbf{x}} \cdot \mathbf{s}_{2}\right)\left(\dot{\theta} \cdot \mathbf{s}_{1}\right)-\left(\dot{\mathbf{x}} \cdot \mathbf{s}_{1}\right)\left(\dot{\theta} \cdot \mathbf{s}_{2}\right) \\
= & \frac{\partial^{2} \phi}{\partial n \partial t} \cdot \mathbf{n}+((\dot{\mathbf{x}} \cdot \nabla) \nabla \phi) \cdot \mathbf{n}+\left(\left(\dot{\mathbf{x}} \cdot \mathbf{s}_{2}\right)-\frac{1}{h_{s_{2}}} \frac{\partial \phi}{\partial s_{2}}\right)\left(\dot{\theta} \cdot \mathbf{s}_{1}\right) \\
& -\left(\left(\dot{\mathbf{x}} \cdot \mathbf{s}_{1}\right)-\frac{1}{h_{s_{1}}} \frac{\partial \phi}{\partial s_{1}}\right)\left(\dot{\theta} \cdot \mathbf{s}_{2}\right)
\end{aligned}
$$

The advection term can be written, using the body condition, as:

$$
\begin{aligned}
(\dot{\mathbf{x}} \cdot \nabla) \nabla \phi= & \left(\dot{\mathbf{x}} \cdot \mathbf{s}_{1}\right) \frac{1}{h_{s_{1}}} \frac{\partial}{\partial s_{1}}\left(\frac{1}{h_{s_{1}}} \frac{\partial \phi}{\partial s_{1}} \mathbf{s}_{1}+\frac{1}{h_{s_{2}}} \frac{\partial \phi}{\partial s_{2}} \mathbf{s}_{2}+\frac{1}{h_{n}} \frac{\partial \phi}{\partial n} \mathbf{n}\right) \\
& +\left(\dot{\mathbf{x}} \cdot \mathbf{s}_{2}\right) \frac{1}{h_{s_{2}}} \frac{\partial}{\partial s_{2}}\left(\frac{1}{h_{s_{1}}} \frac{\partial \phi}{\partial s_{1}} \mathbf{s}_{1}+\frac{1}{h_{s_{2}}} \frac{\partial \phi}{\partial s_{2}} \mathbf{s}_{2}+\frac{1}{h_{n}} \frac{\partial \phi}{\partial n} \mathbf{n}\right) \\
& +(\dot{\mathbf{x}} \cdot \mathbf{n}) \frac{1}{h_{n}} \frac{\partial}{\partial n}\left(\frac{1}{h_{s_{1}}} \frac{\partial \phi}{\partial s_{1}} \mathbf{s}_{1}+\frac{1}{h_{s_{2}}} \frac{\partial \phi}{\partial s_{2}} \mathbf{s}_{2}+\frac{1}{h_{n}} \frac{\partial \phi}{\partial n} \mathbf{n}\right)
\end{aligned}
$$


and thus:

$$
\begin{aligned}
((\dot{\mathbf{x}} \cdot \nabla) \nabla \phi) \cdot \mathbf{n}= & \left(\dot{\mathbf{x}} \cdot \mathbf{s}_{1}\right) \frac{1}{h_{s_{1}}} \frac{1}{h_{n}}\left(\frac{\partial^{2} \phi}{\partial s_{1} \partial n}-\frac{1}{h_{s_{1}}} \frac{\partial h_{s_{1}}}{\partial n} \frac{\partial \phi}{\partial s_{1}}\right) \\
& +\left(\dot{\mathbf{x}} \cdot \mathbf{s}_{2}\right) \frac{1}{h_{s_{2}}} \frac{1}{h_{n}}\left(\frac{\partial^{2} \phi}{\partial s_{2} \partial n}-\frac{1}{h_{s_{2}}} \frac{\partial h_{s_{2}}}{\partial n} \frac{\partial \phi}{\partial s_{2}}\right) \\
& +(\dot{\mathbf{x}} \cdot \mathbf{n}) \frac{1}{h_{n}^{2}} \frac{\partial^{2} \phi}{\partial n^{2}}
\end{aligned}
$$

The Laplace equation gives an expression for the second-order normal derivative of the velocity potential

$$
\frac{1}{h_{n}^{2}} \frac{\partial^{2} \phi}{\partial n^{2}}=-\frac{1}{h_{s_{1}}^{2}} \frac{\partial^{2} \phi}{\partial s_{1}^{2}}-\frac{1}{h_{s_{2}}^{2}} \frac{\partial^{2} \phi}{\partial s_{2}^{2}}-\frac{1}{h_{n}^{2}}\left(\frac{1}{h_{s_{1}}} \frac{\partial h_{s_{1}}}{\partial n}+\frac{1}{h_{s_{2}}} \frac{\partial h_{s_{2}}}{\partial n}\right) \frac{\partial \phi}{\partial n}
$$

Using this in Eq.(38) then yields:

$$
\begin{aligned}
\ddot{\mathbf{x}} \cdot \mathbf{n}= & \frac{\partial^{2} \phi}{\partial n \partial t}+\left(\left(\dot{\mathbf{x}} \cdot \mathbf{s}_{2}\right)-\frac{1}{h_{s_{2}}} \frac{\partial \phi}{\partial s_{2}}\right)\left(\dot{\theta} \cdot \mathbf{s}_{1}\right)-\left(\left(\dot{\mathbf{x}} \cdot \mathbf{s}_{1}\right)-\frac{1}{h_{s_{1}}} \frac{\partial \phi}{\partial s_{1}}\right)\left(\dot{\theta} \cdot \mathbf{s}_{2}\right) \\
& +\left(\dot{\mathbf{x}} \cdot \mathbf{s}_{1}\right) \frac{1}{h_{s_{1}}} \frac{1}{h_{n}}\left(\frac{\partial^{2} \phi}{\partial s_{1} \partial n}-\frac{1}{h_{s_{1}}} \frac{\partial h_{s_{1}}}{\partial n} \frac{\partial \phi}{\partial s_{1}}\right) \\
& +\left(\dot{\mathbf{x}} \cdot \mathbf{s}_{2}\right) \frac{1}{h_{s_{2}}} \frac{1}{h_{n}}\left(\frac{\partial^{2} \phi}{\partial s_{2} \partial n}-\frac{1}{h_{s_{2}}} \frac{\partial h_{s_{2}}}{\partial n} \frac{\partial \phi}{\partial s_{2}}\right) \\
& +(\dot{\mathbf{x}} \cdot \mathbf{n}) \frac{1}{h_{n}^{2}}\left(-\frac{1}{h_{s_{1}}^{2}} \frac{\partial^{2} \phi}{\partial s_{1}^{2}}-\frac{1}{h_{s_{2}}^{2}} \frac{\partial^{2} \phi}{\partial s_{2}^{2}}-\frac{1}{h_{n}^{2}}\left(\frac{1}{h_{s_{1}}} \frac{\partial h_{s_{1}}}{\partial n}+\frac{1}{h_{s_{2}}} \frac{\partial h_{s_{2}}}{\partial n}\right) \frac{\partial \phi}{\partial n}\right)
\end{aligned}
$$

Finally, using the scale factors and their derivatives, we obtain:

$$
\begin{aligned}
\frac{\partial^{2} \phi}{\partial n \partial t}=\ddot{\mathbf{x}} \cdot \mathbf{n} & +\left(\dot{\theta} \cdot \mathbf{s}_{1}\right)\left(\frac{\partial \phi}{\partial s_{2}}-\left(\dot{\mathbf{x}} \cdot \mathbf{s}_{2}\right)\right)-\left(\dot{\theta} \cdot \mathbf{s}_{2}\right)\left(\frac{\partial \phi}{\partial s_{1}}-\left(\dot{\mathbf{x}} \cdot \mathbf{s}_{1}\right)\right) \\
& +\left(\dot{\mathbf{x}} \cdot \mathbf{s}_{1}\right)\left(\frac{1}{R_{1}} \frac{\partial \phi}{\partial s_{1}}-\frac{\partial^{2} \phi}{\partial s_{1} \partial n}\right)+\left(\dot{\mathbf{x}} \cdot \mathbf{s}_{2}\right)\left(\frac{1}{R_{2}} \frac{\partial \phi}{\partial s_{2}}-\frac{\partial^{2} \phi}{\partial s_{2} \partial n}\right) \\
& +(\dot{\mathbf{x}} \cdot \mathbf{n})\left(\frac{\partial^{2} \phi}{\partial s_{1}^{2}}+\frac{\partial^{2} \phi}{\partial s_{2}^{2}}+\left(\frac{1}{R_{1}}+\frac{1}{R_{2}}\right) \frac{\partial \phi}{\partial n}\right)
\end{aligned}
$$

In order to get the van Daalen expression, Eq.(12), we only need to consider opposite signs in front of $\frac{1}{R_{1}}$ and $\frac{1}{R_{2}}$, since the normal vectors are opposite in van Daalen(1993).

\subsection{Tanizawa expression}

Tanizawa derived a different 2D expression for the body boundary condition, based on the acceleration of a fluid particle sliding on the body surface. The material derivative associated to that fluid particle motion is:

$$
\frac{D}{D t}=\frac{\partial}{\partial t}+\nabla \phi \cdot \nabla
$$




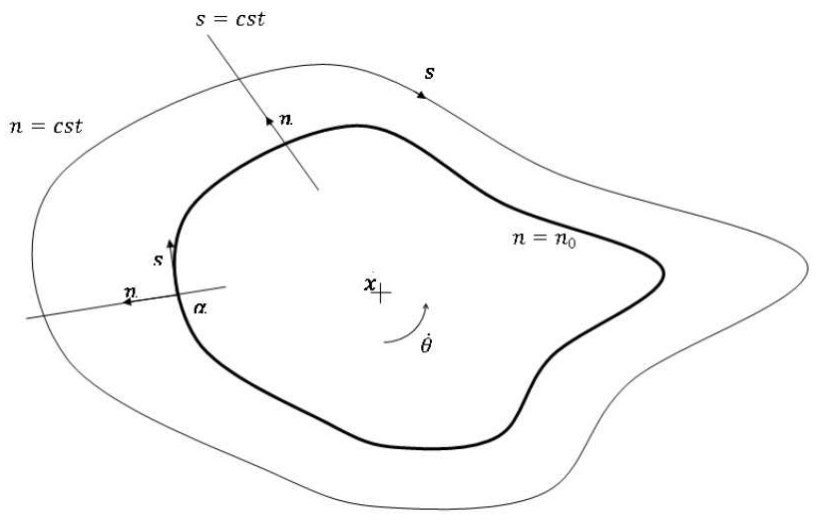

(a) 2D Tanizawa basis

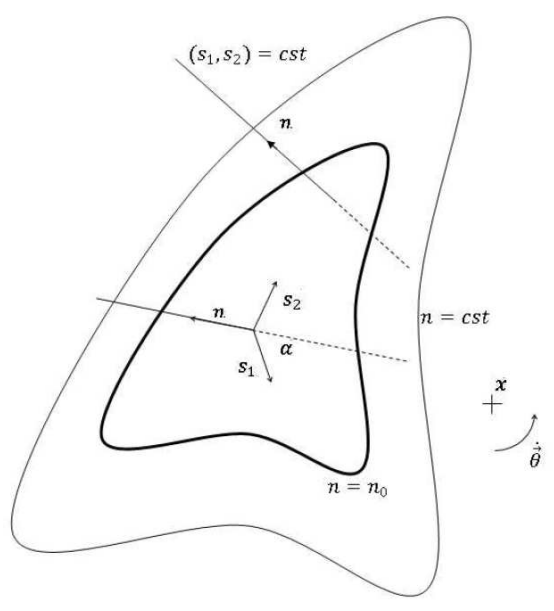

(b) 3D Berkvens basis

Figure 5: bases used by Tanizawa and Berkvens

The acceleration of the fluid particle can be written as:

$$
\mathbf{a}=\frac{D \mathbf{v}}{D t}=\frac{D \nabla \phi}{D t}=\frac{\partial \nabla \phi}{\partial t}+(\nabla \phi \cdot \nabla) \nabla \phi=\nabla \frac{\partial \phi}{\partial t}+(\nabla \phi \cdot \nabla) \nabla \phi
$$

Its normal component is then:

$$
\frac{\partial^{2} \phi}{\partial n \partial t}=\mathbf{a} \cdot \mathbf{n}-(\nabla \phi \cdot \nabla) \nabla \phi \cdot \mathbf{n}
$$

The second term on the right hand side of the previous equation was developed by Tanizawa Tanizawa(1995)] in 2D, as follows:

$$
(\nabla \phi \cdot \nabla) \nabla \phi \cdot \mathbf{n}=-\frac{1}{R}\left(\left(\frac{\partial \phi}{\partial s}\right)^{2}+\left(\frac{\partial \phi}{\partial n}\right)^{2}\right)+\frac{\partial \phi}{\partial s} \frac{\partial^{2} \phi}{\partial s \partial n}-\frac{\partial \phi}{\partial n} \frac{\partial^{2} \phi}{\partial s^{2}}
$$

Tanizawa used a 2D direct basis, with a normal vector defined pointing outside the body (see Fig. 5a).

It was later extended to 3D by Berkvens [Berkvens(1998), Tanizawa(2000), with a similar basis, direct and normal pointing outside the body (see Fig. 5b):

$$
\begin{aligned}
(\nabla \phi \cdot \nabla) \nabla \phi \cdot \mathbf{n}= & -\left(\frac{1}{R_{1}}+\frac{1}{R_{2}}\right)\left(\frac{\partial \phi}{\partial n}\right)^{2}-\frac{1}{R_{1}}\left(\frac{\partial \phi}{\partial s_{1}}\right)^{2}-\frac{1}{R_{2}}\left(\frac{\partial \phi}{\partial s_{2}}\right)^{2} \\
& -\left(\frac{\partial^{2} \phi}{\partial s_{1}^{2}}+\frac{\partial^{2} \phi}{\partial s_{2}^{2}}\right) \frac{\partial \phi}{\partial n}+\frac{\partial \phi}{\partial s_{1}} \frac{\partial^{2} \phi}{\partial s_{1} \partial n}+\frac{\partial \phi}{\partial s_{2}} \frac{\partial^{2} \phi}{\partial s_{2} \partial n}
\end{aligned}
$$




\subsubsection{D Expression}

Using the 2D curvilinear basis previously introduced, Fig. 3, it is possible to write the velocity potential gradient as:

leading to:

$$
\nabla \phi=\frac{1}{h_{s}} \frac{\partial \phi}{\partial s} \mathbf{s}+\frac{1}{h_{n}} \frac{\partial \phi}{\partial n} \mathbf{n}
$$

$$
\begin{aligned}
(\nabla \phi \cdot \nabla)(\nabla \phi)= & \frac{1}{h_{s}} \frac{\partial \phi}{\partial s} \frac{1}{h_{s}} \frac{\partial}{\partial s}\left(\frac{1}{h_{s}} \frac{\partial \phi}{\partial s} \mathbf{s}+\frac{1}{h_{n}} \frac{\partial \phi}{\partial n} \mathbf{n}\right) \\
& +\frac{1}{h_{n}} \frac{\partial \phi}{\partial n} \frac{1}{h_{n}} \frac{\partial}{\partial n}\left(\frac{1}{h_{s}} \frac{\partial \phi}{\partial s} \mathbf{s}+\frac{1}{h_{n}} \frac{\partial \phi}{\partial n} \mathbf{n}\right) \\
= & \frac{1}{h_{s}^{2}} \frac{\partial \phi}{\partial s}\left[\frac{1}{h_{s}} \frac{\partial^{2} \phi}{\partial s^{2}} \mathbf{s}-\frac{1}{h_{s}} \frac{\partial \phi}{\partial s} \frac{1}{h_{n}} \frac{\partial h_{s}}{\partial n} \mathbf{n}+\frac{1}{h_{n}} \frac{\partial^{2} \phi}{\partial s \partial n} \mathbf{n}+\frac{1}{h_{n}} \frac{\partial \phi}{\partial n} \frac{1}{h_{n}} \frac{\partial h_{s}}{\partial n} \mathbf{s}\right] \\
& +\frac{1}{h_{n}^{2}} \frac{\partial \phi}{\partial n}\left[\frac{1}{h_{s}} \frac{\partial^{2} \phi}{\partial n \partial s} \mathbf{s}+\frac{1}{h_{n}} \frac{\partial^{2} \phi}{\partial n^{2}} \mathbf{n}\right]
\end{aligned}
$$

Using Laplace equation to remove the second order normal derivative, the normal component is then:

$$
\begin{aligned}
(\nabla \phi \cdot \nabla)(\nabla \phi) \cdot \mathbf{n}= & -\frac{1}{h_{s}^{3}} \frac{1}{h_{n}} \frac{\partial h_{s}}{\partial n}\left(\frac{\partial \phi}{\partial s}\right)^{2}+\frac{1}{h_{s}^{2}} \frac{1}{h_{n}} \frac{\partial \phi}{\partial s} \frac{\partial^{2} \phi}{\partial s \partial n}+\frac{1}{h_{n}^{3}} \frac{\partial \phi}{\partial n} \frac{\partial^{2} \phi}{\partial n^{2}} \\
= & -\frac{1}{h_{s}^{3}} \frac{1}{h_{n}} \frac{\partial h_{s}}{\partial n}\left(\frac{\partial \phi}{\partial s}\right)^{2}+\frac{1}{h_{s}^{2}} \frac{1}{h_{n}} \frac{\partial \phi}{\partial s} \frac{\partial^{2} \phi}{\partial s \partial n} \\
& -\frac{1}{h_{n}} \frac{\partial \phi}{\partial n}\left(\frac{1}{h_{s}^{2}} \frac{\partial^{2} \phi}{\partial s^{2}}+\frac{1}{h_{s}} \frac{\partial h_{s}}{\partial n} \frac{\partial \phi}{\partial n}\right) \\
= & -\frac{1}{h_{s} h_{n}} \frac{\partial h_{s}}{\partial n}\left(\frac{1}{h_{s}^{2}}\left(\frac{\partial \phi}{\partial s}\right)^{2}+\left(\frac{\partial \phi}{\partial n}\right)^{2}\right)+\frac{1}{h_{s}^{2}} \frac{1}{h_{n}} \frac{\partial \phi}{\partial s} \frac{\partial^{2} \phi}{\partial s \partial n} \\
& -\frac{1}{h_{s}^{2}} \frac{1}{h_{n}} \frac{\partial \phi}{\partial n} \frac{\partial^{2} \phi}{\partial s^{2}}
\end{aligned}
$$

Using the scale factors $h_{s}=h_{n}=1$ and their derivatives $\frac{1}{h_{s}} \frac{\partial h_{s}}{\partial n}=\frac{1}{R}$, the previous equation yields the Tanizawa expression, Eq.(46):

$$
(\nabla \phi \cdot \nabla)(\nabla \phi) \cdot \mathbf{n}=-\frac{1}{R}\left(\left(\frac{\partial \phi}{\partial s}\right)^{2}+\left(\frac{\partial \phi}{\partial n}\right)^{2}\right)+\frac{\partial \phi}{\partial s} \frac{\partial^{2} \phi}{\partial s \partial n}-\frac{\partial \phi}{\partial n} \frac{\partial^{2} \phi}{\partial s^{2}}
$$

\subsubsection{D expression}

The 3D curvilinear basis previously introduced, Fig. 4, is also used to describe the velocity potential gradient:

$$
\nabla \phi=\frac{1}{h_{s_{1}}} \frac{\partial \phi}{\partial s_{1}} \mathbf{s}_{1}+\frac{1}{h_{s_{2}}} \frac{\partial \phi}{\partial s_{2}} \mathbf{s}_{2}+\frac{1}{h_{n}} \frac{\partial \phi}{\partial n} \mathbf{n}
$$


yielding:

$$
\begin{aligned}
(\nabla \phi \cdot \nabla) \nabla \phi= & \frac{1}{h_{s_{1}^{2}}} \frac{\partial \phi}{\partial s_{1}} \frac{\partial}{\partial s_{1}}\left(\frac{1}{h_{s_{1}}} \frac{\partial \phi}{\partial s_{1}} \mathbf{s}_{1}+\frac{1}{h_{s_{2}}} \frac{\partial \phi}{\partial s_{2}} \mathbf{s}_{2}+\frac{1}{h_{n}} \frac{\partial \phi}{\partial n} \mathbf{n}\right) \\
& +\frac{1}{h_{s_{2}^{2}}} \frac{\partial \phi}{\partial s_{2}} \frac{\partial}{\partial s_{2}}\left(\frac{1}{h_{s_{1}}} \frac{\partial \phi}{\partial s_{1}} \mathbf{s}_{1}+\frac{1}{h_{s_{2}}} \frac{\partial \phi}{\partial s_{2}} \mathbf{s}_{2}+\frac{1}{h_{n}} \frac{\partial \phi}{\partial n} \mathbf{n}\right) \\
& +\frac{1}{h_{n}^{2}} \frac{\partial \phi}{\partial n} \frac{\partial}{\partial n}\left(\frac{1}{h_{s_{1}}} \frac{\partial \phi}{\partial s_{1}} \mathbf{s}_{1}+\frac{1}{h_{s_{2}}} \frac{\partial \phi}{\partial s_{2}} \mathbf{s}_{2}+\frac{1}{h_{n}} \frac{\partial \phi}{\partial n} \mathbf{n}\right)
\end{aligned}
$$

The normal component is then:

$$
\begin{aligned}
((\nabla \phi \cdot \nabla) \nabla \phi) \cdot \mathbf{n}= & \frac{1}{h_{s_{1}^{2}}} \frac{1}{h_{n}} \frac{\partial \phi}{\partial s_{1}}\left(\frac{\partial^{2} \phi}{\partial s_{1} \partial n}-\frac{1}{h_{s_{1}}} \frac{\partial h_{s_{1}}}{\partial n} \frac{\partial \phi}{\partial s_{1}}\right) \\
& +\frac{1}{h_{s_{2}^{2}}} \frac{1}{h_{n}} \frac{\partial \phi}{\partial s_{2}}\left(\frac{\partial^{2} \phi}{\partial s_{2} \partial n}-\frac{1}{h_{s_{2}}} \frac{\partial h_{s_{1}}}{\partial n} \frac{\partial \phi}{\partial s_{1}}\right) \\
& +\frac{1}{h_{n}^{3}} \frac{\partial \phi}{\partial n} \frac{\partial^{2} \phi}{\partial n^{2}}
\end{aligned}
$$

The second-order normal derivative of the velocity potential is given by the Laplace equation. Using the scale factors and their derivatives, we obtain Berkvens' expression, Eq.(47):

$$
\begin{aligned}
((\nabla \phi \cdot \nabla) \nabla \phi) \cdot \mathbf{n}= & \frac{\partial \phi}{\partial s_{1}}\left(\frac{\partial^{2} \phi}{\partial s_{1} \partial n}-\frac{1}{R_{1}} \frac{\partial \phi}{\partial s_{1}}\right)+\frac{\partial \phi}{\partial s_{2}}\left(\frac{\partial^{2} \phi}{\partial s_{2} \partial n}-\frac{1}{R_{2}} \frac{\partial \phi}{\partial s_{2}}\right) \\
& +\frac{\partial \phi}{\partial n}\left(-\frac{\partial^{2} \phi}{\partial s_{1}^{2}}-\frac{\partial^{2} \phi}{\partial s_{2}^{2}}-\left(\frac{1}{R_{1}}+\frac{1}{R_{2}}\right) \frac{\partial \phi}{\partial n}\right) \\
= & -\left(\frac{1}{R_{1}}+\frac{1}{R_{2}}\right)\left(\frac{\partial \phi}{\partial n}\right)^{2}-\frac{1}{R_{1}}\left(\frac{\partial \phi}{\partial s_{1}}\right)^{2}-\frac{1}{R_{2}}\left(\frac{\partial \phi}{\partial s_{2}}\right)^{2} \\
& -\frac{\partial \phi}{\partial n}\left(\frac{\partial^{2} \phi}{\partial s_{1}^{2}}+\frac{\partial^{2} \phi}{\partial s_{2}^{2}}\right)+\frac{\partial \phi}{\partial s_{1}} \frac{\partial^{2} \phi}{\partial s_{1} \partial n}+\frac{\partial \phi}{\partial s_{2}} \frac{\partial^{2} \phi}{\partial s_{2} \partial n}
\end{aligned}
$$

\subsection{Equivalence of the expressions}

Both expressions are implemented in different potential flow solvers, to simulate nonlinear body motions, and were subject of validations. Cointe expression was implemented by Cointe Cointe(1989)], Guerber [Guerber et al.(2012)Guerber, Benoit, Grilli, and Buvat and van Daalen [van Daalen(1993)], while Tanizawa expression was implemented by Tanizawa [Tanizawa(1995)], Berkvens [Berkvens(1998)] and Koo [Koo and Kim(2004)]. However the theoretical equivalence of these expression has not been shown yet.

\subsubsection{D Expressions}

In order to demonstrate the equivalence between the Cointe and Tanizawa expressions, it is necessary to express the acceleration of the fluid particle sliding on the body surface 
in terms of the acceleration of the related point on the body. The coordinates of these two points are $\mathbf{x}$ in the global coordinate system and $\mathbf{r}$ in the local one related to the body. The position, velocity and acceleration vectors of the point on the body surface can be written as:

$$
\left\{\begin{array}{l}
\mathbf{x}=\mathbf{x}_{b}+\mathbf{r} \\
\dot{\mathbf{x}}=\dot{\mathbf{x}}_{b}+\dot{\theta} \wedge \mathbf{r} \\
\ddot{\mathbf{x}}=\ddot{\mathbf{x}}_{b}+\ddot{\theta} \wedge \mathbf{r}+\dot{\theta} \wedge \dot{\theta} \wedge \mathbf{r}
\end{array}\right.
$$

where $\left(\mathbf{x}_{b}, \dot{\mathbf{x}}_{b}, \ddot{\mathbf{x}}_{b}\right)$ are the position, velocity and acceleration of the center of gravity of the body, $\mathbf{x}=X \mathbf{e}_{x}+Y \mathbf{e}_{y}$ and $\theta=\theta \mathbf{e}_{z}$.

The local coordinates of the point on the body surface, $\mathbf{r}$, are constant in time since the local coordinate system follows the body motion, which means $\dot{\mathbf{r}}=0$. This is however not the case for the fluid particle, of velocity $\mathbf{v}$ and acceleration $\mathbf{a}$ :

$$
\left\{\begin{array}{l}
\mathbf{x}=\mathbf{x}_{b}+\mathbf{r} \\
\mathbf{v}=\dot{\mathbf{x}}_{b}+\dot{\theta} \wedge \mathbf{r}+\dot{\mathbf{r}}=\dot{\mathbf{x}}+\dot{\mathbf{r}} \\
\mathbf{a}=\ddot{\mathbf{x}}_{b}+\ddot{\theta} \wedge \mathbf{r}+\dot{\theta} \wedge \dot{\theta} \wedge \mathbf{r}+2 \dot{\theta} \wedge \dot{\mathbf{r}}+\ddot{\mathbf{r}}=\ddot{\mathbf{x}}+2 \dot{\theta} \wedge \dot{\mathbf{r}}+\ddot{\mathbf{r}}
\end{array}\right.
$$

The time derivative of the local coordinates of the fluid particle can then be written, making use of the free slip condition on the body, as:

$$
\dot{\mathbf{r}}=\mathbf{v}-\dot{\mathbf{x}}=\nabla \phi-\dot{\mathbf{x}}=\left(\frac{\partial \phi}{\partial s}-(\dot{\mathbf{x}} \cdot \mathbf{s})\right) \mathbf{s}
$$

Tanizawa gave an expression for $\ddot{\mathbf{r}} \cdot \mathbf{n}$ based on the derivative in the Frenet-Serret frame [Tanizawa(1995)]:

$$
\ddot{\mathbf{r}} \cdot \mathbf{n}=-k|\dot{\mathbf{r}}|^{2}=-\frac{1}{R}\left(\frac{\partial \phi}{\partial s}-(\dot{\mathbf{x}} \cdot \mathbf{s})\right)^{2}
$$

The fluid particle is indeed sliding on the body surface, following a parametric curve, as illustrated in Fig. 6.

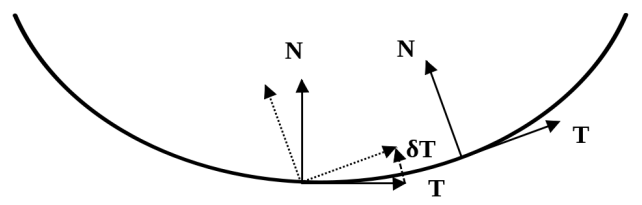

Figure 6: Illustration of the Frenet-Serret frame

The position of the particle is given by $\mathbf{r}(s, t)=(x(s, t), y(s, t))$. The tangent and normal vectors are defined, for any point $\mathbf{x}(s, t)$ on the parametric curve, as:

$$
\mathbf{T}=\frac{\partial \mathbf{x}(s, t)}{\partial s}=\left(\frac{\partial X}{\partial s}, \frac{\partial Y}{\partial s}\right) \quad \text { and } \quad \mathbf{N}=-R \frac{\partial \mathbf{T}}{\partial s}
$$

where $R$ is the local curvature radius. 
The particle's velocity on the curve can then be written, using the scalar velocity $\mathbf{v}=\frac{\partial \mathbf{s}}{\partial t}$, as:

$$
\dot{\mathbf{r}}(s, t)=\left(\frac{\partial X}{\partial s} \cdot \frac{\partial \mathbf{s}}{\partial t}, \frac{\partial Y}{\partial s} \cdot \frac{\partial \mathbf{s}}{\partial t}\right)=\mathbf{v} \mathbf{T}=|\dot{\mathbf{r}}(s, t)| \mathbf{T}
$$

Similarly, the acceleration is:

$$
\begin{gathered}
\ddot{\mathbf{r}}(s, t)=\frac{\partial|\dot{\mathbf{r}}(s, t)|}{\partial t} \mathbf{T}+|\dot{\mathbf{r}}(s, t)| \frac{\partial \mathbf{T}}{\partial t}=\frac{\partial|\dot{\mathbf{r}}(s, t)|}{\partial t} \mathbf{T}+|\dot{\mathbf{r}}(s, t)| \frac{\partial \mathbf{T}}{\partial s} \frac{\partial s}{\partial t} \\
\ddot{\mathbf{r}}(s, t)=\frac{\partial|\dot{\mathbf{r}}(s, t)|}{\partial t} \mathbf{T}-\frac{|\dot{\mathbf{r}}(s, t)|^{2}}{R} \mathbf{N}
\end{gathered}
$$

Finally, the normal component of acceleration is:

$$
\ddot{\mathbf{r}}(s, t) \cdot \mathbf{n}=-\frac{1}{R}|\dot{\mathbf{r}}|^{2}=-\frac{1}{R}\left(\frac{\partial \phi}{\partial s}-(\dot{\mathbf{x}} \cdot \mathbf{s})\right)^{2}
$$

It is then possible to relate the normal acceleration of a fluid particle on the boundary $\mathbf{a} \cdot \mathbf{n}$ to the one associated to the body $\ddot{\mathbf{x}} \cdot \mathbf{n}$ :

$$
\mathbf{a} \cdot \mathbf{n}=\ddot{\mathbf{x}} \cdot \mathbf{n}+2 \dot{\theta}\left(\frac{\partial \phi}{\partial s}-(\dot{\mathbf{x}} \cdot \mathbf{s})\right)-\frac{1}{R}\left(\frac{\partial \phi}{\partial s}-(\dot{\mathbf{x}} \cdot \mathbf{s})\right)^{2}
$$

Introducing this relation into the Tanizawa expression, Eq.(46), the normal derivative of the time derivative of the velocity potential can be written as:

$$
\begin{aligned}
\frac{\partial^{2} \phi}{\partial n \partial t}= & \mathbf{a} \cdot \mathbf{n}-(\nabla \phi \cdot \nabla) \nabla \phi \cdot \mathbf{n} \\
= & \ddot{\mathbf{x}} \cdot \mathbf{n}+2 \dot{\theta}\left(\frac{\partial \phi}{\partial s}-(\dot{\mathbf{x}} \cdot \mathbf{s})\right)-\frac{1}{R}\left(\frac{\partial \phi}{\partial s}-(\dot{\mathbf{x}} \cdot \mathbf{s})\right)^{2}+\frac{1}{R}\left(\left(\frac{\partial \phi}{\partial s}\right)^{2}+\left(\frac{\partial \phi}{\partial n}\right)^{2}\right) \\
& -\frac{\partial \phi}{\partial s} \frac{\partial^{2} \phi}{\partial s \partial n}+\frac{\partial \phi}{\partial n} \frac{\partial^{2} \phi}{\partial s^{2}} \\
= & \ddot{\mathbf{x}} \cdot \mathbf{n}+2 \dot{\theta}\left(\frac{\partial \phi}{\partial s}-(\dot{\mathbf{x}} \cdot \mathbf{s})\right)-\frac{1}{R}(\dot{\mathbf{x}} \cdot \mathbf{s})^{2}+\frac{2}{R}(\dot{\mathbf{x}} \cdot \mathbf{s}) \frac{\partial \phi}{\partial s}-\frac{\partial \phi}{\partial s} \frac{\partial^{2} \phi}{\partial s \partial n} \\
& +\frac{\partial \phi}{\partial n}\left(\frac{\partial^{2} \phi}{\partial s^{2}}+\frac{1}{R} \frac{\partial \phi}{\partial n}\right) \\
= & \ddot{\mathbf{x}} \cdot \mathbf{n}+\dot{\theta}\left(\frac{\partial \phi}{\partial s}-(\dot{\mathbf{x}} \cdot \mathbf{s})\right)-(\dot{\mathbf{x}} \cdot \mathbf{s})\left(\frac{\partial^{2} \phi}{\partial s \partial n}-\frac{1}{R} \frac{\partial \phi}{\partial s}\right)+(\dot{\mathbf{x}} \cdot \mathbf{n})\left(\frac{\partial^{2} \phi}{\partial s^{2}}+\frac{1}{R} \frac{\partial \phi}{\partial n}\right) \\
& +\dot{\theta}\left(\frac{\partial \phi}{\partial s}-(\dot{\mathbf{x}} \cdot \mathbf{s})\right)-\frac{1}{R}(\dot{\mathbf{x}} \cdot \mathbf{s})^{2}+\frac{1}{R}(\dot{\mathbf{x}} \cdot \mathbf{s}) \frac{\partial \phi}{\partial s}-\frac{\partial \phi}{\partial s} \frac{\partial^{2} \phi}{\partial s \partial n}+(\dot{\mathbf{x}} \cdot \mathbf{s}) \frac{\partial^{2} \phi}{\partial s \partial n} \\
= & \ddot{\mathbf{x}} \cdot \mathbf{n}+\dot{\theta}\left(\frac{\partial \phi}{\partial s}-(\dot{\mathbf{x}} \cdot \mathbf{s})\right)+(\dot{\mathbf{x}} \cdot \mathbf{s})\left(\frac{1}{R} \frac{\partial \phi}{\partial s}-\frac{\partial^{2} \phi}{\partial s \partial n}\right)+(\dot{\mathbf{x}} \cdot \mathbf{n})\left(\frac{\partial^{2} \phi}{\partial s^{2}}+\frac{1}{R} \frac{\partial \phi}{\partial n}\right) \\
& +\left(\dot{\theta}-\frac{\partial^{2} \phi}{\partial s \partial n}+\frac{1}{R}(\dot{\mathbf{x}} \cdot \mathbf{s})\right)\left(\frac{\partial \phi}{\partial s}-(\dot{\mathbf{x}} \cdot \mathbf{s})\right)
\end{aligned}
$$


The tangential derivative of the normal derivative of the velocity potential can be written, using the body condition, as:

$$
\begin{aligned}
\frac{\partial^{2} \phi}{\partial s \partial n} & =\frac{\partial}{\partial s}\left(\frac{\partial \phi}{\partial n}\right)=\frac{\partial}{\partial s}(\dot{\mathbf{x}} \cdot \mathbf{n}) \\
& =\frac{\partial \dot{\mathbf{x}}}{\partial s} \cdot \mathbf{n}+\dot{\mathbf{x}} \cdot \frac{\partial \mathbf{n}}{\partial s} \\
& =\frac{\partial\left(\dot{\mathbf{x}}_{b}+\dot{\theta} \mathbf{e}_{z} \wedge \mathbf{r}\right)}{\partial s} \cdot \mathbf{n}+\frac{1}{R}(\dot{\mathbf{x}} \cdot \mathbf{s}) \\
& =\left(\dot{\theta} \mathbf{e}_{z} \wedge \frac{\partial \mathbf{r}}{\partial s}\right) \cdot \mathbf{n}+\frac{1}{R}(\dot{\mathbf{x}} \cdot \mathbf{s}) \\
& =\dot{\theta}\left(\mathbf{e}_{z} \wedge \mathbf{s}\right) \cdot \mathbf{n}+\frac{1}{R}(\dot{\mathbf{x}} \cdot \mathbf{s})
\end{aligned}
$$

leading to:

$$
\dot{\theta}-\frac{\partial^{2} \phi}{\partial s \partial n}+\frac{1}{R}(\dot{\mathbf{x}} \cdot \mathbf{s})=0
$$

Introducing this relation to Eq.(66) yields the Cointe expression, Eq.(29).

\subsubsection{D Expressions}

The fluid particle acceleration can be written, in 3D, using the related body point acceleration, in the same manner as in $2 \mathrm{D}$, as

$$
\mathbf{a} \cdot \mathbf{n}=\ddot{\mathbf{x}} \cdot \mathbf{n}+(2 \dot{\theta} \wedge \dot{\mathbf{r}}) \cdot \mathbf{n}+\ddot{\mathbf{r}} \cdot \mathbf{n}
$$

where:

$$
(\dot{\theta} \wedge \dot{\mathbf{r}}) \cdot \mathbf{n}=\left(\dot{\theta} \cdot \mathbf{s}_{1}\right)\left(\frac{\partial \phi}{\partial s_{2}}-\dot{\mathbf{x}} \cdot \mathbf{s}_{2}\right)-\left(\dot{\theta} \cdot \mathbf{s}_{2}\right)\left(\frac{\partial \phi}{\partial s_{1}}-\dot{\mathbf{x}} \cdot \mathbf{s}_{1}\right)
$$

and:

$$
\ddot{\mathbf{r}} \cdot \mathbf{n}=-\frac{1}{R_{1}}\left(\frac{\partial \phi}{\partial s_{1}}-\dot{\mathbf{x}} \cdot \mathbf{s}_{1}\right)^{2}-\frac{1}{R_{2}}\left(\frac{\partial \phi}{\partial s_{2}}-\dot{\mathbf{x}} \cdot \mathbf{s}_{2}\right)^{2}
$$

Introducing the previous equations into Berkvens' expression, Eq.(47), the normal 
component of the time derivative of the velocity potential can be written as:

$$
\begin{aligned}
\frac{\partial^{2} \phi}{\partial n \partial t}= & \mathbf{a} \cdot \mathbf{n}-((\nabla \phi \cdot \nabla) \nabla \phi) \cdot \mathbf{n} \\
= & \ddot{\mathbf{x}} \cdot \mathbf{n}+2\left(\dot{\theta} \cdot \mathbf{s}_{1}\right)\left(\frac{\partial \phi}{\partial s_{2}}-\dot{\mathbf{x}} \cdot \mathbf{s}_{2}\right)-2\left(\dot{\theta} \cdot \mathbf{s}_{2}\right)\left(\frac{\partial \phi}{\partial s_{1}}-\dot{\mathbf{x}} \cdot \mathbf{s}_{1}\right) \\
& -\frac{1}{R_{1}}\left(\frac{\partial \phi}{\partial s_{1}}-\dot{\mathbf{x}} \cdot \mathbf{s}_{1}\right)^{2}-\frac{1}{R_{2}}\left(\frac{\partial \phi}{\partial s_{2}}-\dot{\mathbf{x}} \cdot \mathbf{s}_{2}\right)^{2}+\left(\frac{1}{R_{1}}+\frac{1}{R_{2}}\right)\left(\frac{\partial \phi}{\partial n}\right)^{2} \\
& +\frac{1}{R_{1}}\left(\frac{\partial \phi}{\partial s_{1}}\right)^{2}+\frac{1}{R_{1}}\left(\frac{\partial \phi}{\partial s_{2}}\right)^{2}+\frac{\partial \phi}{\partial n}\left(\frac{\partial^{2} \phi}{\partial s_{1}^{2}}+\frac{\partial^{2} \phi}{\partial s_{2}^{2}}\right)-\frac{\partial \phi}{\partial s_{1}} \frac{\partial^{2} \phi}{\partial s_{1} \partial n}-\frac{\partial \phi}{\partial s_{2}} \frac{\partial^{2} \phi}{\partial s_{2} \partial n} \\
= & \ddot{\mathbf{x}} \cdot \mathbf{n}+2\left(\dot{\theta} \cdot \mathbf{s}_{1}\right)\left(\frac{\partial \phi}{\partial s_{2}}-\dot{\mathbf{x}} \cdot \mathbf{s}_{2}\right)-2\left(\dot{\theta} \cdot \mathbf{s}_{2}\right)\left(\frac{\partial \phi}{\partial s_{1}}-\dot{\mathbf{x}} \cdot \mathbf{s}_{1}\right) \\
& -\frac{1}{R_{1}}\left(\dot{\mathbf{x}} \cdot \mathbf{s}_{1}\right)^{2}+\frac{2}{R_{1}}\left(\dot{\mathbf{x}} \cdot \mathbf{s}_{1}\right) \frac{\partial \phi}{\partial s_{1}}-\frac{1}{R_{2}}\left(\dot{\mathbf{x}} \cdot \mathbf{s}_{2}\right)^{2}+\frac{2}{R_{2}}\left(\dot{\mathbf{x}} \cdot \mathbf{s}_{2}\right) \frac{\partial \phi}{\partial s_{2}} \\
& +(\dot{\mathbf{x}} \cdot \mathbf{n})\left(\frac{\partial^{2} \phi}{\partial s_{1}^{2}}+\frac{\partial^{2} \phi}{\partial s_{2}^{2}}+\left(\frac{1}{R_{1}}+\frac{1}{R_{2}}\right) \frac{\partial \phi}{\partial n}\right)-\frac{\partial \phi}{\partial s_{1}} \frac{\partial^{2} \phi}{\partial s_{1} \partial n}-\frac{\partial \phi}{\partial s_{2}} \frac{\partial^{2} \phi}{\partial s_{2} \partial n}
\end{aligned}
$$

leading to:

$$
\begin{aligned}
\frac{\partial^{2} \phi}{\partial n \partial t}= & \ddot{\mathbf{x}} \cdot \mathbf{n}+\left(\dot{\theta} \cdot \mathbf{s}_{1}\right)\left(\frac{\partial \phi}{\partial s_{2}}-\left(\dot{\mathbf{x}} \cdot \mathbf{s}_{2}\right)\right)-\left(\dot{\theta} \cdot \mathbf{s}_{2}\right)\left(\frac{\partial \phi}{\partial s_{1}}-\left(\dot{\mathbf{x}} \cdot \mathbf{s}_{1}\right)\right) \\
+ & \left(\dot{\mathbf{x}} \cdot \mathbf{s}_{1}\right)\left(\frac{1}{R_{1}} \frac{\partial \phi}{\partial s_{1}}-\frac{\partial^{2} \phi}{\partial s_{1} \partial n}\right)+\left(\dot{\mathbf{x}} \cdot \mathbf{s}_{2}\right)\left(\frac{1}{R_{2}} \frac{\partial \phi}{\partial s_{2}}-\frac{\partial^{2} \phi}{\partial s_{2} \partial n}\right) \\
& +(\dot{\mathbf{x}} \cdot \mathbf{n})\left(\frac{\partial^{2} \phi}{\partial s_{1}^{2}}+\frac{\partial^{2} \phi}{\partial s_{2}^{2}}+\left(\frac{1}{R_{1}}+\frac{1}{R_{2}}\right) \frac{\partial \phi}{\partial n}\right) \\
& -\left(\frac{\partial \phi}{\partial s_{1}}-\left(\dot{\mathbf{x}} \cdot \mathbf{s}_{1}\right)\right)\left[\frac{\partial^{2} \phi}{\partial s_{1} \partial n}-\frac{1}{R_{1}}\left(\dot{\mathbf{x}} \cdot \mathbf{s}_{1}\right)+\left(\dot{\theta} \cdot \mathbf{s}_{2}\right)\right] \\
& -\left(\frac{\partial \phi}{\partial s_{2}}-\left(\dot{\mathbf{x}} \cdot \mathbf{s}_{2}\right)\right)\left[\frac{\partial^{2} \phi}{\partial s_{2} \partial n}-\frac{1}{R_{2}}\left(\dot{\mathbf{x}} \cdot \mathbf{s}_{2}\right)-\left(\dot{\theta} \cdot \mathbf{s}_{1}\right)\right]
\end{aligned}
$$

The tangential derivatives of the normal velocities can be written in the same manner as in $2 \mathrm{D}$ :

$$
\left\{\begin{array}{l}
\frac{\partial^{2} \phi}{\partial s_{1} \partial n}-\frac{1}{R_{1}}\left(\dot{\mathbf{x}} \cdot \mathbf{s}_{1}\right)+\left(\dot{\theta} \cdot \mathbf{s}_{\mathbf{2}}\right)=0 \\
\frac{\partial^{2} \phi}{\partial s_{2} \partial n}-\frac{1}{R_{2}}\left(\dot{\mathbf{x}} \cdot \mathbf{s}_{2}\right)-\left(\dot{\theta} \cdot \mathbf{s}_{\mathbf{1}}\right)=0
\end{array}\right.
$$

Using these relations in Eq.(172) leads to the van Daalen expression, Eq.(42).

\subsection{Unified expression}

A unified expression is proposed below for a direct local basis with a normal vector pointing outside the body. From a numerical perspective, this simplifies the use of Cointe 


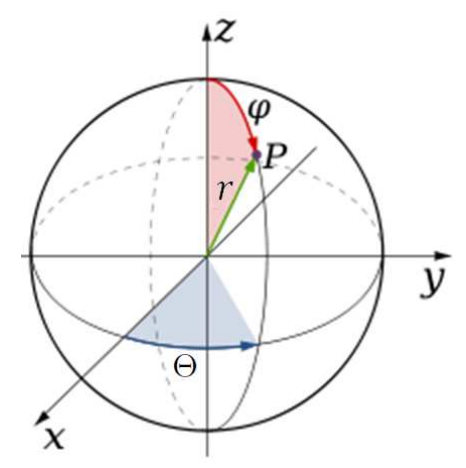

Figure 7: Spherical coordinates

expression. Indeed, Cointe expression requires the evaluation of the local derivatives of the normal velocity $\frac{\partial^{2} \phi}{\partial s \partial n}$. They must be approximated numerically, which is a source of inaccuracy. By using Eq.(67) and Eq.(73), the tangential derivatives of the normal velocity are replaced with known variables that can be evaluated without any further approximation. The proposed unified expression reads in $2 \mathrm{D}$ :

$$
\begin{aligned}
\frac{\partial^{2} \phi}{\partial n \partial t}= & \ddot{\mathbf{x}} \cdot \mathbf{n}+\dot{\theta}\left(\frac{\partial \phi}{\partial s}-2(\dot{\mathbf{x}} \cdot \mathbf{s})\right)+\frac{(\dot{\mathbf{x}} \cdot \mathbf{s})}{R}\left(\frac{\partial \phi}{\partial s}-(\dot{\mathbf{x}} \cdot \mathbf{s})\right) \\
& +(\dot{\mathbf{x}} \cdot \mathbf{n})\left(\frac{\partial^{2} \phi}{\partial s^{2}}+\frac{1}{R} \frac{\partial \phi}{\partial n}\right)
\end{aligned}
$$

and in 3D

$$
\begin{aligned}
\frac{\partial^{2} \phi}{\partial n \partial t} & =\ddot{\mathbf{x}} \cdot \mathbf{n}+\left(\dot{\theta} \cdot \mathbf{s}_{1}\right)\left(\frac{\partial \phi}{\partial s_{2}}-2\left(\dot{\mathbf{x}} \cdot \mathbf{s}_{2}\right)\right)-\left(\dot{\theta} \cdot \mathbf{s}_{2}\right)\left(\frac{\partial \phi}{\partial s_{1}}-2\left(\dot{\mathbf{x}} \cdot \mathbf{s}_{1}\right)\right) \\
& +\frac{\left(\dot{\mathbf{x}} \cdot \mathbf{s}_{1}\right)}{R_{1}}\left(\frac{\partial \phi}{\partial s_{1}}-\left(\dot{\mathbf{x}} \cdot \mathbf{s}_{1}\right)\right)+\frac{\left(\dot{\mathbf{x}} \cdot \mathbf{s}_{2}\right)}{R_{2}}\left(\frac{\partial \phi}{\partial s_{2}}-\left(\dot{\mathbf{x}} \cdot \mathbf{s}_{2}\right)\right) \\
& +(\dot{\mathbf{x}} \cdot \mathbf{n})\left(\frac{\partial^{2} \phi}{\partial s_{1}^{2}}+\frac{\partial^{2} \phi}{\partial s_{2}^{2}}+\left(\frac{1}{R_{1}}+\frac{1}{R_{2}}\right) \frac{\partial \phi}{\partial n}\right)
\end{aligned}
$$

\section{APPLICATION TO A SIMPLE CASE}

\subsection{Analytical solution for a sphere in prescribed motions in an un- bounded fluid domain}

We consider a sphere in an unbounded fluid domain, of radius $a$, in spherical coordinates $(r, \Theta, \psi)$, as defined in Fig. 7

$$
\left\{\begin{array}{l}
x=r \sin (\Theta) \cos (\psi) \\
y=r \sin (\Theta) \sin (\psi) \\
z=r \cos (\Theta)
\end{array}\right.
$$


The sphere velocity is forced to $\mathbf{V}=U \mathbf{e}_{z}=A_{u} \sin (\omega t) \mathbf{e}_{z}$.

The local curvilinear coordinates can be defined on the sphere as:

$$
\left\{\begin{array} { l } 
{ s _ { 1 } = r \Theta } \\
{ s _ { 2 } = r \psi } \\
{ n = r }
\end{array} \Leftrightarrow \left\{\begin{array}{rl}
\Theta & =\frac{s_{1}}{r} \\
\psi & =\frac{s_{2}}{r} \\
r & =n
\end{array}\right.\right.
$$

The following identity can then be obtained:

$$
\left\{\begin{array}{l}
U \cos (\Theta)=\mathbf{V} \cdot \mathbf{n} \\
U \sin (\Theta)=-\mathbf{V} \cdot \mathbf{s}_{1}
\end{array}\right.
$$

An analytical solution for the velocity potential in the sphere reference system has been given by Lamb $[\mathrm{Lamb}(1993)]$ for this particular case:

$$
\phi(r, \Theta, \psi)=-\frac{1}{2} U \frac{a^{3}}{r^{2}} \cos (\Theta)
$$

On the sphere, the velocity potential can be written, for $r=a$ :

$$
\phi(r=a, \Theta)=-\frac{1}{2} U a \cos (\Theta)=-\frac{1}{2} a \mathbf{V} \cdot \mathbf{n}
$$

The local derivatives, expressed using the local curvilinear coordinates, are:

$$
\left\{\begin{array}{l}
\frac{\partial \phi}{\partial s_{1}}=\frac{\partial \phi}{\partial r} \frac{\partial r}{\partial s_{1}}+\frac{\partial \phi}{\partial \Theta} \frac{\partial \Theta}{\partial s_{1}}+\frac{\partial \phi}{\partial \psi} \frac{\partial \psi}{\partial s_{1}}=\frac{1}{2} U \frac{a^{3}}{r^{3}} \sin (\Theta) \\
\frac{\partial \phi}{\partial s_{2}}=\frac{\partial \phi}{\partial r} \frac{\partial r}{\partial s_{2}}+\frac{\partial \phi}{\partial \Theta} \frac{\partial \Theta}{\partial s_{2}}+\frac{\partial \phi}{\partial \phi} \frac{\partial \psi}{\partial s_{2}}=0 \\
\frac{\partial \phi}{\partial n}=\frac{\partial \phi}{\partial r}=U \frac{a^{3}}{r^{3}} \cos (\Theta)
\end{array}\right.
$$

which yields on the sphere:

$$
\left\{\begin{array}{l}
\frac{\partial \phi}{\partial s_{1}}=\frac{1}{2} U \sin (\Theta)=-\frac{1}{2} \mathbf{V} \cdot \mathbf{s}_{1} \\
\frac{\partial \phi}{\partial s_{2}}=0 \\
\frac{\partial \phi}{\partial n}=\mathbf{V} \cdot \mathbf{n}
\end{array}\right.
$$

The gradient can then be expressed as:

$$
\nabla \phi=U \frac{a^{3}}{r^{3}} \cos (\Theta) \mathbf{n}+\frac{1}{2} U \frac{a^{3}}{r^{3}} \sin (\Theta) \mathbf{s}_{1}=\frac{a^{3}}{r^{3}}(\mathbf{V} \cdot \mathbf{n}) \mathbf{n}-\frac{1}{2} \frac{a^{3}}{r^{3}}\left(\mathbf{V} \cdot \mathbf{s}_{1}\right) \mathbf{s}_{1}
$$

The velocity potential is expressed in the reference coordinates associated to the motions of the sphere. The time derivative of the expression of the velocity potential is 
then a material derivative. The partial derivative of the velocity potential can thus be written as:

$$
\frac{\partial \phi}{\partial t}=\frac{D \phi}{D t}-\mathbf{V} \cdot \nabla \phi=-\frac{1}{2} \frac{a^{3}}{r^{2}}(\mathbf{A} \cdot \mathbf{n})+\frac{1}{2} \frac{a^{3}}{r^{3}}\left(\mathbf{V} \cdot \mathbf{s}_{1}\right)^{2}-\frac{a^{3}}{r^{3}}(\mathbf{V} \cdot \mathbf{n})^{2}
$$

where $\mathbf{A}=A_{u} \omega \cos (\omega t) \mathbf{z}$ is the body acceleration.

The partial time derivative of the velocity potential on the body is then:

$$
\frac{\partial \phi}{\partial t}(r=a)=-\frac{1}{2} a(\mathbf{A} \cdot \mathbf{n})-\frac{1}{2}\left(\mathbf{V} \cdot \mathbf{s}_{1}\right)^{2}-(\mathbf{V} \cdot \mathbf{n})^{2}
$$

It is possible to get the normal derivative of the time derivative of the velocity potential, from the previous equations, as:

$$
\frac{\partial^{2} \phi}{\partial n \partial t}=\frac{\partial^{2} \phi}{\partial r \partial t}=\frac{a^{3}}{r^{3}}(\mathbf{A} \cdot \mathbf{n})-\frac{3}{2} \frac{a^{3}}{r^{4}}\left(\mathbf{V} \cdot \mathbf{s}_{1}\right)^{2}+3 \frac{a^{3}}{r^{4}}(\mathbf{V} \cdot \mathbf{n})^{2}
$$

which leads, on the body, to:

$$
\frac{\partial^{2} \phi}{\partial n \partial t}(r=a)=(\mathbf{A} \cdot \mathbf{n})-\frac{3}{2 a}\left(\mathbf{V} \cdot \mathbf{s}_{1}\right)^{2}+\frac{3}{a}(\mathbf{V} \cdot \mathbf{n})^{2}
$$

\subsection{Unified expression for this case}

In this particular case, the unified expression for the body boundary condition Eq.(75) yields:

$$
\begin{aligned}
\frac{\partial^{2} \phi}{\partial n \partial t}= & (\mathbf{A} \cdot \mathbf{n})+\frac{\left(\mathbf{V} \cdot \mathbf{s}_{1}\right)}{a}\left(\frac{\partial \phi}{\partial s_{1}}-\left(\mathbf{V} \cdot \mathbf{s}_{1}\right)\right)+\frac{\left(\mathbf{V} \cdot \mathbf{s}_{2}\right)}{a}\left(\frac{\partial \phi}{\partial s_{2}}-\left(\mathbf{V} \cdot \mathbf{s}_{2}\right)\right) \\
& +(\mathbf{V} \cdot \mathbf{n})\left(\frac{\partial^{2} \phi}{\partial s_{1}^{2}}+\frac{\partial^{2} \phi}{\partial s_{2}^{2}}+\frac{2}{a} \frac{\partial \phi}{\partial n}\right)
\end{aligned}
$$

The Laplace equation for the velocity potential in curvilinear coordinates is:

$$
\triangle \phi=\frac{\partial^{2} \phi}{\partial s_{1}^{2}}+\frac{\partial^{2} \phi}{\partial s_{2}^{2}}+\frac{\partial^{2} \phi}{\partial n^{2}}+\frac{2}{a} \frac{\partial \phi}{\partial n}=0
$$

so the unified expression can be written as:

$$
\frac{\partial^{2} \phi}{\partial n \partial t}=(\mathbf{A} \cdot \mathbf{n})+\frac{\left(\mathbf{V} \cdot \mathbf{s}_{1}\right)}{a}\left(\frac{\partial \phi}{\partial s_{1}}-\left(\mathbf{V} \cdot \mathbf{s}_{1}\right)\right)+\frac{\left(\mathbf{V} \cdot \mathbf{s}_{2}\right)}{a}\left(\frac{\partial \phi}{\partial s_{2}}-\left(\mathbf{V} \cdot \mathbf{s}_{2}\right)\right)-(\mathbf{V} \cdot \mathbf{n}) \frac{\partial^{2} \phi}{\partial n^{2}}
$$

This last second order derivative can be expressed in curvilinear coordinates:

$$
\frac{\partial^{2} \phi}{\partial n^{2}}=\frac{\partial^{2} \phi}{\partial r^{2}}=-3 U \frac{a^{3}}{r^{4}} \cos (\Theta)
$$

which yields on the body

$$
\frac{\partial^{2} \phi}{\partial n^{2}}(r=a)=-\frac{3 U}{a} \cos (\Theta)=-\frac{3}{a}(\mathbf{V} \cdot \mathbf{n})
$$


The unified expression can then be written as:

$$
\frac{\partial^{2} \phi}{\partial n \partial t}=(\mathbf{A} \cdot \mathbf{n})-\frac{3}{2 a}\left(\mathbf{V} \cdot \mathbf{s}_{1}\right)^{2}+\frac{3}{a}(\mathbf{V} \cdot \mathbf{n})^{2}
$$

The unified expression gives the same result as the analytical one. It is then possible to conclude on the validity of the unified expression in this simple case. The numerical implementation of this unified expression in potential flow solvers can then be used to simulate more complex cases : complex body geometries, rotational motions, etc.

\section{CONCLUSION}

The simulation of freely moving bodies in potential flow theory requires an accurate evaluation of the hydrodynamic force. Its accuracy depends mainly on the correct calculation of the time derivative of the velocity potential on the body. While a finite difference scheme can be used for bodies in prescribed motions, it is not accurate enough for freely moving bodies [Cointe(1989), Koo and Kim(2004)] with explicit time-stepping schemes. Several methods have been proposed to evaluate this time derivative, some requiring solving a BVP for the time derivative of the velocity potential. While the free-surface conditions can be obtained easily, the body condition requires a careful treatment. Two sets of equations have been given (for 2D and 3D), first by Cointe [Cointe(1989)] (2D) and van Daalen van Daalen(1993) (3D), using the acceleration of a point on the body and the other ones by Tanizawa [Tanizawa(1995)] (2D) and Berkvens [Berkvens(1998)] (3D), using the acceleration of a fluid particle sliding on the body. These expressions were developed for different notations and bases, direct or indirect, some with a normal vector pointing inside the body and others outside the body, not fully explicited in the references.

The first objective of this study was to check each expression, by redeveloping them using a direct curvilinear basis with a normal vector pointing outside the body. The four expressions were obtained successfully, and the differences due to the basis originally used were explained. The equivalence of 2D and 3D expressions was proven, by expressing the acceleration of a fluid particle sliding on the body using the acceleration of its associated point on the body. The demonstration highlighted a particular identity giving an expression of local derivatives of the normal velocity using known variables. A unified expression could thus be given, simplifying Cointe and van Daalen expressions, by using said identity. The main advantage of this unified expression over Cointe-van Daalen ones lies in the fact that the local derivatives of the normal velocity does not need to be computed, leading to an increased accuracy of this evaluation.

Finally, the unified expression was validated against an analytical case, consisting of a submerged sphere in an unbounded fluid domain with prescribed motion. The body condition was developed analytically and compared to the analytical developments of the unified expression. The same results could be obtained in both cases, validating the unified expression in this case. 
This new expression of the body condition can then be implemented in a potential flow solver, in order to simulate free body motions, including bodies with more complex geometry. Of particular interest to the authors are wave energy converters with large amplitude motions [Letournel et al.(tted)Letournel, Chauvigné, Gelly, Babarit, Ducrozet, and Ferrant].

\section{ACKNOWLEDGMENT}

The authors thank ANR (Agence Nationale de la Recherche) for their financial support of this work as part of the project ANR11-MONU-018-01 MONACOREV [Monacorev(2012)].

\section{BIBLIOGRAPHY}

\section{References}

[Cointe(1989)] R. Cointe, Quelques aspects de la simulation numérique d'un canal à houle, Ph.D. thesis, École Nationale des Ponts et Chaussées, 1989.

[van Daalen(1993)] E. F. G. van Daalen, Numerical and Theoretical Studies of Water Waves and Floating Bodies, Ph.D. thesis, University of Twente, The Netherlands, 1993.

[Tanizawa(1995)] K. Tanizawa, A Nonlinear Simulation Method of 3-D Body Motions in Waves (1st Report), Journal of the Society of Naval Architects of Japan 1995 (178) (1995) 179-191.

[Berkvens(1998)] P. J. F. Berkvens, Floating bodies interacting with water waves: Development of a time-domain panel method, Ph.D. thesis, Universiteit Twente (The Netherlands), 1998.

[Longuet-Higgins and Cokelet(1976)] M. S. Longuet-Higgins, E. D. Cokelet, The Deformation of Steep Surface Waves on Water. I. A Numerical Method of Computation, Proceedings of the Royal Society of London. A. Mathematical and Physical Sciences 350 (1660) (1976) $1-26$.

[Xü, H Yue(1992)] D. Xü, H Yue, Numerical study of three dimensional overturning waves, in: Proc. 7th Intl. Workshop on Water Waves and Floating Bodies, 303-307, 1992.

[Dommermuth and Yue(1987)] D. Dommermuth, D. Yue, Numerical simulations of nonlinear axisymmetric flows with a free surface, Journal of Fluid Mechanics .

[Zhang et al.(1996)Zhang, Yue, and Tanizawa] S. Zhang, D. Yue, K. Tanizawa, Simulation of plunging wave impact on a vertical wall, Journal of Fluid . 
[Grilli et al.(2000)Grilli, Guyenne, and Dias] S. Grilli, P. Guyenne, F. Dias, Modeling of overturning waves over arbitrary bottom in a 3D numerical wave tank, The Tenth International Offshore and .

[Ferrant(1998)] P. Ferrant, Fully Non-Linear Interactions of Long-Crested Wave Packets with a Three -Dimensional Body, in: 22nd ONR Symposium on Naval Hydrodynamics, 403-415, 1998.

[Kashiwagi(1996)] M. Kashiwagi, Full-nonlinear simulations of hydrodynamic forces on a heaving two-dimensional body, Journal of the Society of Naval Architects of Japan

[Koo and Kim(2004)] W. Koo, M.-H. Kim, Freely floating-body simulation by a 2D fully nonlinear numerical wave tank, Ocean Engineering 31 (16) (2004) 2011-2046, ISSN 00298018.

[Vinje and Brevig(1981)] T. Vinje, P. Brevig, Numerical simulation of breaking waves, Advances in Water Resources .

[Cointe and Geyer(1990)] R. Cointe, P. Geyer, Nonlinear and linear motions of a rectangular barge in a perfect fluid, in: 18th Symposium on Naval Hydrodynamics, Ann Arbor, MI, USA, 85, 1990.

[Sen(1993)] D. Sen, Numerical simulation of motions of two-dimensional floating bodies, Journal of Ship Research 37 (4) (1993) 307.

[Cao et al.(1994)Cao, Beck, and Schultz] Y. Cao, R. Beck, W. Schultz, Nonlinear computation of wave loads and motions of floating bodies in incident waves, on Water Waves and Floating Bodies ( . . . .

[Wu and Eatock Taylor(1996)] G. Wu, R. Eatock Taylor, Transient motion of a floating body in steep water waves, in: Proceedings of the 11th International Workshop on Water Waves and Floating Bodies (IWWWFB), -, 1996.

[Tanizawa(2000)] K. Tanizawa, The state of the art on numerical wave tank, in: 4th Osaka colloquium on seakeeping performance ships, 95-114, 2000.

[Guerber(2011)] E. Guerber, Modélisation numérique des intéractions non-linéaires entre vagues et structures immergées, appliquée à la simulation de systèmes houlomoteurs, Ph.D. thesis, Unversité Paris-Est, 2011.

[Newman(1977)] J. N. Newman, Marine Hydrodynamics, MIT Press, 1977.

[Wehausen and Laitone(1960)] J. Wehausen, E. Laitone, Surface Waves, Springer, 1960.

[Guerber et al.(2012)Guerber, Benoit, Grilli, and Buvat] E. Guerber, M. Benoit, S. T. Grilli, C. Buvat, A fully nonlinear implicit model for wave interactions with submerged structures in forced or free motion, Engineering Analysis with Boundary Elements 36 (7) (2012) 1151-1163, ISSN 09557997. 
[Lamb(1993)] H. Lamb, Hydrodynamics, Cambridge Mathematical Library, Cambridge University Press, Cambridge, 1993.

[Letournel et al.(tted)Letournel, Chauvigné, Gelly, Babarit, Ducrozet, and Ferrant] L. Letournel, C. Chauvigné, B. Gelly, A. Babarit, G. Ducrozet, P. Ferrant, Weakly Non linear Modeling of Submerged Wave Energy Converters, Applied Ocean Research Submitted.

[Monacorev(2012)] Monacorev, URL http://lheea.ec-nantes.fr/doku.php/monacorev/partenaires, 2012. 